\title{
Computing dynamical degrees of rational maps on moduli space
}

\author{
SARAH KOCH $\dagger$ and ROLAND K. W. ROEDER $\$$ \\ $\dagger$ University of Michigan, East Hall, 530 Church Street, Ann Arbor, MI 48109, USA \\ (e-mail: kochsc@umich.edu) \\ $\ddagger$ IUPUI, Department of Mathematical Sciences, LD Building, Room 270, \\ 402 North Blackford Street, Indianapolis, IN 46202-3267, USA \\ (e-mail: rroeder@math.iupui.edu)
}

(Received 20 August 2014 and accepted in revised form 11 March 2015)

\begin{abstract}
The dynamical degrees of a rational map $f: X \rightarrow X$ are fundamental invariants describing the rate of growth of the action of iterates of $f$ on the cohomology of $X$. When $f$ has non-empty indeterminacy set, these quantities can be very difficult to determine. We study rational maps $f: X^{N} \rightarrow X^{N}$, where $X^{N}$ is isomorphic to the Deligne-Mumford compactification $\overline{\mathcal{M}}_{0, N+3}$. We exploit the stratified structure of $X^{N}$ to provide new examples of rational maps, in arbitrary dimension, for which the action on cohomology behaves functorially under iteration. From this, all dynamical degrees can be readily computed (given enough book-keeping and computing time). In this paper, we explicitly compute all of the dynamical degrees for all such maps $f: X^{N} \rightarrow X^{N}$, where $\operatorname{dim}\left(X^{N}\right) \leqslant 3$ and the first dynamical degrees for the mappings where $\operatorname{dim}\left(X^{N}\right) \leqslant 5$. These examples naturally arise in the setting of Thurston's topological characterization of rational maps.
\end{abstract}

\section{Introduction}

Let $X$ be a smooth, compact, complex algebraic variety of dimension $N$. A rational map $f: X \rightarrow X$ induces a pullback action $f^{*}: H^{k, k}(X ; \mathbb{C}) \rightarrow H^{k, k}(X ; \mathbb{C})$ (defined in $\left.\S 2\right)$. A typical starting point for studying the dynamics associated to iterating $f$ is to compute the dynamical degrees

$$
\lambda_{k}(f):=\lim _{n \rightarrow \infty}\left\|\left(f^{n}\right)^{*}: H^{k, k}(X ; \mathbb{C}) \rightarrow H^{k, k}(X ; \mathbb{C})\right\|^{1 / n},
$$

which are defined for $0 \leqslant k \leqslant N$. Given the dynamical degrees of $f$, there is a precise description of what ergodic properties $f$ should have; see, for example, [Gu1]. These properties have been established when $\lambda_{N}(f)$ is maximal [Gu2, DNT] or when $\operatorname{dim}(X)=2, \lambda_{1}(f)>\lambda_{2}(f)$, and certain minor technical hypotheses are satisfied [DDG]. 
Dynamical degrees were originally introduced by Friedland $[\mathbf{F r}]$ and later by Russakovskii and Shiffman [RS] and shown to be invariant under birational conjugacy by Dinh and Sibony [DS2]. Dynamical degrees were originally defined with a lim sup instead of a limit in equation (1) above; however, it was shown in [DS2, DS1] that the limit always exists.

If the map $f$ has points of indeterminacy, then the iterates of $f$ may not act functorially on $H^{k, k}(X ; \mathbb{C})$, which can be a formidable obstacle to computing the dynamical degree $\lambda_{k}(f)$. If the action of $f^{*}$ on $H^{k, k}(X ; \mathbb{C})$ is functorial, that is, for all $m>0$,

$$
\left(f^{*}\right)^{m}: H^{k, k}(X ; \mathbb{C}) \rightarrow H^{k, k}(X ; \mathbb{C}) \quad \text { equals } \quad\left(f^{m}\right)^{*}: H^{k, k}(X ; \mathbb{C}) \rightarrow H^{k, k}(X ; \mathbb{C}),
$$

then the map $f: X \rightarrow X$ said to be $k$-stable. In this case, it immediately follows that the dynamical degree $\lambda_{k}(f)$ is the spectral radius of $f^{*}: H^{k, k}(X ; \mathbb{C}) \rightarrow H^{k, k}(X ; \mathbb{C})$. If $f: X \rightarrow X$ is $k$-stable for all $1 \leqslant k \leqslant N$, then the map $f: X \rightarrow X$ is said to be algebraically stable. Note that $f^{*}$ is automatically functorial on $H^{N, N}(X ; \mathbb{C})$ and $\lambda_{N}(f)$ is the topological degree of $f$. For more background and discussion of dynamical degrees and algebraic stability, we refer the reader to $[\mathbf{B}, \mathbf{R}]$.

Given an arbitrary map $f: X \rightarrow X$, the problem of verifying that $f$ is algebraically stable (or modifying $X$ in order to conjugate $f$ to an algebraically stable map) can be quite subtle, as is the problem of determining all of the dynamical degrees $\lambda_{1}(f), \lambda_{2}(f), \ldots, \lambda_{N}(f)$. The purpose of this paper is to study these problems for a specific family of maps $f_{\rho}: X^{N} \rightarrow X^{N}$ where both the map $f_{\rho}$ and the space $X^{N}$ have additional structure.

More specifically, the space $X^{N}$ will be isomorphic to $\overline{\mathcal{M}}_{0, n}$, the Deligne-Mumford compactification of $\mathcal{M}_{0, n}$, where $\mathcal{M}_{0, n}$ is the moduli space of genus 0 curves with $n$ labeled points. The space $\overline{\mathcal{M}}_{0, n}$ is a smooth projective variety of dimension $N=$ $n-3[\mathbf{K n}, \mathbf{K M}]$. Given a permutation $\rho \in S_{n}$, we build a map $f_{\rho}:=g_{\rho} \circ s: X^{N} \rightarrow X^{N}$, where $s: X^{N} \rightarrow X^{N}$ is a relatively simple map to understand (although it has a nonempty indeterminacy set), and $g_{\rho}: X^{N} \rightarrow X^{N}$ is an automorphism of $X^{N}$ induced by the permutation $\rho$. The resulting $f_{\rho}$ has topological degree $\lambda_{N}\left(f_{\rho}\right)=2^{N}$, so it remains to consider the other dynamical degrees $\lambda_{k}\left(f_{\rho}\right)$ for $1 \leqslant k<N$.

We can prove stability in a somewhat wider context: for a fixed $N \geqslant 1$, let $\mathcal{F}^{N}$ denote the semi-group of rational self-mappings of $X^{N}$ generated under composition by all of the mappings of the form $f_{\rho}: X^{N} \rightarrow X^{N}$. The following theorem is our first main result.

THEOREM 1.1. For any fixed $N \geqslant 1$ the semi-group $\mathcal{F}^{N}$ acts functorially on all cohomology groups of $X^{N}$, that is, for any $f_{1}, f_{2} \in \mathcal{F}^{N}$ and any $1 \leqslant k \leqslant N$ we have

$$
\left(f_{1} \circ f_{2}\right)^{*}=f_{2}^{*} \circ f_{1}^{*}: H^{k, k}\left(X^{N} ; \mathbb{C}\right) \rightarrow H^{k, k}\left(X^{N} ; \mathbb{C}\right) .
$$

The following corollary is immediate.

COROLlARY 1.2. For any $N \geqslant 1$, any $f \in \mathcal{F}^{N}$ is an algebraically stable self-map of $X^{N}$. In particular, for any $1 \leqslant k \leqslant N$ we have that $\lambda_{k}(f)$ equals the spectral radius of

$$
f^{*}: H^{k, k}\left(X^{N} ; \mathbb{C}\right) \rightarrow H^{k, k}\left(X^{N} ; \mathbb{C}\right) .
$$


TABLE 1. Table of dimensions of $H^{k, k}\left(X^{N}\right)$.

\begin{tabular}{cccccccc}
\hline & \multicolumn{7}{c}{$k$} \\
\cline { 2 - 7 }$N$ & 0 & 1 & 2 & 3 & 4 & 5 & 6 \\
\hline 0 & 1 & & & & & & \\
1 & 1 & 1 & & & & & \\
2 & 1 & 5 & 1 & & & & \\
3 & 1 & 16 & 16 & 1 & & & \\
4 & 1 & 42 & 127 & 42 & 1 & & \\
5 & 1 & 99 & 715 & 715 & 99 & 1 & \\
6 & 1 & 219 & 3292 & 7723 & 3292 & 219 & 1 \\
\hline
\end{tabular}

For the remainder of the paper we focus on computing the dynamical degrees of the generators $f_{\rho}$ of $\mathcal{F}^{N}$. By Corollary 1.2 they should be easy to compute. However, we are confronted with another challenge: the dimension of $H^{k, k}\left(X^{N} ; \mathbb{C}\right)$ grows exponentially with $N$ (see Table 1). These numbers were computed using a theorem of Keel (Theorem 3.4 below, published in $[\mathbf{K e}]$ ) which provides generators and relations for the cohomology ring $H^{*}\left(X^{N} ; \mathbb{C}\right)$. Keel's theorem will play a central role in all of our calculations. Computing $\left(f_{\rho}\right)^{*}$ on $H^{k, k}\left(X^{N} ; \mathbb{C}\right)$ is rather difficult because $\operatorname{dim}\left(H^{k, k}\left(X^{n} ; \mathbb{C}\right)\right)$ is large.

Our second main result is that we provide an algorithmic approach to computing $\left(f_{\rho}\right)^{*}: H^{1,1}(X ; \mathbb{C}) \rightarrow H^{1,1}(X ; \mathbb{C})$, which is presented in $\S 6$. This allows us to readily compute $\lambda_{1}\left(f_{\rho}\right)$ for any $N$ and $\rho$, using the computer algebra system Sage (http: / / www. sagemath.org). Values of $\lambda_{1}\left(f_{\rho}\right)$ for $N=2,3,4,5$ and various $\rho$ are tabulated in $\S 7$.

It is far more technical to compute $\left(f_{\rho}\right)^{*}$ for $k \geqslant 2$ because a subvariety $V \subset X^{N}$ of codimension greater than or equal to 2 may have preimage $f^{-1}(V)$ lying entirely in the indeterminacy set $I\left(f_{\rho}\right)$. Our final main result is computation of

$$
\left(f_{\rho}\right)^{*}: H^{2,2}\left(X^{3} ; \mathbb{C}\right) \rightarrow H^{2,2}\left(X^{3} ; \mathbb{C}\right)
$$

in $\S 6$. The resulting values for $\lambda_{2}\left(f_{\rho}\right)$ are tabulated in $\S 7$. With sufficient book-keeping, we expect that this can be done for all $N$ and $k$.

Remark 1.3. For a given $\rho$, the space $X^{N}$ may not be optimal, meaning that there is a space $Z^{N}$, obtained by blowing down certain hypersurfaces, on which (a conjugate of) $f_{\rho}$ is still algebraically stable. (For example, certain choices of $\rho$, including $\rho=\mathrm{id}$, result in a mapping $f_{\rho}$ that is algebraically stable on $\mathbb{P}^{N}$.) Similarly, if one is only interested in $k$-stability for a particular value of $k$, there may be a blow-down $Z^{N}$ of $X^{N}$ on which all of the mappings $f_{\rho}$ are $k$-stable (see Remark 7.1). The merit of working with $X^{N}$ is that every mapping $f_{\rho}$ is $k$-stable for all $1 \leqslant k \leqslant N$ on the same space $X^{N}$.

In the literature. Dynamical degrees have been extensively studied for maps $f: X \rightarrow X$ where $X$ is a surface. If $f$ is a bimeromorphic map of a compact Kähler surface, Diller and Favre [DF] proved that there is a proper modification $\pi: \widehat{X} \rightarrow X$ so that $f$ lifts to an algebraically stable map $\widehat{f}: \widehat{X} \rightarrow \widehat{X}$. The space $\widehat{X}$ and lifted map $\widehat{f}$ are 
called a stabilization of $f$. However, in [Fa], Favre found examples of monomial maps $f: \mathbb{P}^{2}-\rightarrow \mathbb{P}^{2}$ of topological degree 2 or greater for which no such stabilization exists.

In the higher-dimensional case $f: X \rightarrow X$, the question of the functoriality of $f^{*}$ on $H^{1,1}(X ; \mathbb{C})$ (i.e. whether or not $f$ is 1 -stable) has been extensively studied [BK1, BK2, BT, JW, HP]. The functoriality of $f^{*}$ on $H^{k, k}(X ; \mathbb{C})$ for $2 \leqslant k \leqslant N-1$ is typically even more delicate. In [Li2], Lin computes all of the dynamical degrees for monomial maps $\mathbb{P}^{3} \rightarrow \mathbb{P}^{3}$. In $[\mathbf{B K 3}, \mathbf{B C K}]$, Bedford and Kim and then Bedford et al study pseudoautomorphisms of three-dimensional manifolds, computing all dynamical degrees for a certain family of such maps. In [Am], Amerik computes all dynamical degrees for a particular map $f: X \rightarrow X$, where $X$ is a four-dimensional smooth compact complex projective variety arising in an algebro-geometric context. Favre and Wulcan $[\mathbf{F a W}]$ and Lin [Li1] compute all dynamical degrees for monomial maps $\mathbb{P}^{n} \rightarrow \mathbb{P}^{n}$, and Lin and Wulcan $[\mathbf{L W}]$ study the problem of stabilizing certain monomial maps $\mathbb{P}^{n} \rightarrow \mathbb{P}^{n}$. There is also a notion of the arithmetic degree (of a point) for dominant rational maps $\mathbb{P}^{n} \rightarrow \mathbb{P}^{n}$ defined in $[\mathbf{S i}]$.

Motivation. The maps $f: X^{N} \rightarrow X^{N}$ in Theorem 1.2 constitute a new family of examples for which algebraic stability is known and for which all of the dynamical degrees can be systematically computed (with enough book-keeping). They also fit nicely within the context of stabilization, since Kapranov's theorem [Ka] expresses $X^{N}$ as an iterated blow-up of the projective space $\mathbb{P}^{N}$. We initially studied (conjugates of) these mappings on $\mathbb{P}^{N}$ and later discovered that all of them stabilize when lifted to $X^{N}$.

Moreover, the maps $f_{\rho}: X^{N} \rightarrow X^{N}$ naturally arise in the setting of Thurston's topological characterization of rational maps [Ko]. As a general rule, dynamical quantities associated to iterating the maps $f_{\rho}$ should correspond to dynamical quantities associated to iterating the Thurston pullback map on a Teichmüller space.

Outline. We begin the paper in $\$ 2$ with some background on the action of a rational map $f: X \rightarrow X$ on cohomology and statement of the criterion for functoriality of a composition (Proposition 2.1) that will be used to proof Theorem 1.1. In $\S 3$ we discuss several important properties of the moduli space $\overline{\mathcal{M}}_{0, n}$, including Keel's theorem and Kapranov's theorem. Basic properties of the mapping $f_{\rho}$ are presented in $\S 4$. Section 5 is dedicated to the proof of Theorem 1.2. Computations of $\left(f_{\rho}\right)^{*}$ are done in $\S 6$. A catalog of dynamical degrees for specific examples is presented in $\S 7$.

\section{Action on cohomology}

We begin by explaining how a dominant rational map $f: X \rightarrow Y$ between smooth complex projective varieties of dimension $N$ induces a well-defined pullback $f^{*}: H^{k, k}(Y ; \mathbb{C}) \rightarrow H^{k, k}(X ; \mathbb{C})$ even though $f$ may have a non-empty indeterminacy set $I_{f}$ (necessarily of codimension 2). We will first work with the singular cohomology $H^{i}(Y ; \mathbb{C}) \rightarrow H^{i}(X ; \mathbb{C})$, and we will then remark about why this definition preserves bidegree.

For the remainder of the paper we will use the term projective manifold to mean smooth, compact, complex projective variety. If $V$ is a $k$-dimensional subvariety of a 
projective manifold $X$ of dimension $N$, then $V$ determines a fundamental homology class $\{V\} \in H_{2 k}(X ; \mathbb{C})$. The fundamental cohomology class of $V$ is $[V]:=\mathrm{PD}^{-1}(\{V\}) \in$ $H^{2 N-2 k}(X ; C)$, where $\mathrm{PD}_{M}: H^{j}(M) \rightarrow H_{\left(\operatorname{dim}_{\mathbb{R}}(M)-j\right)}(M)$ denotes the Poincaré duality isomorphism on a manifold $M$.

Let

$$
\Gamma_{f}=\overline{\left\{(x, y) \in X \times Y: x \notin I_{f} \text { and } y=f(x)\right\}}
$$

be the graph of $f$ and let $\left[\Gamma_{f}\right] \in H^{2 N}(X \times Y ; \mathbb{C})$ denote its fundamental cohomology class. Let $\pi_{1}: X \times Y \rightarrow X$ and $\pi_{2}: X \times Y \rightarrow Y$ denote the canonical projection maps. For any $\alpha \in H^{i}(Y ; \mathbb{C})$, one defines

$$
f^{*} \alpha:=\pi_{1 *}\left(\left[\Gamma_{f}\right] \smile \pi_{2}^{*} \alpha\right) .
$$

Here, $\pi_{2}^{*}$ is the classical pullback on cohomology, as defined for regular maps, and $\pi_{1 *}$ is the pushforward on cohomology, defined by $\pi_{1 *}=\mathrm{PD}_{X}^{-1} \circ \pi_{1 \#} \circ \mathrm{PD}_{X \times Y}$, where $\pi_{1 \#}$ denotes the pushforward on homology. If $f$ is regular (i.e. $I_{f}=\emptyset$ ) then $(2)$ coincides with the classical definition of pullback.

Suppose that there exist a projective manifold $\widetilde{X}$ and holomorphic maps pr and $\tilde{f}$ making the diagram

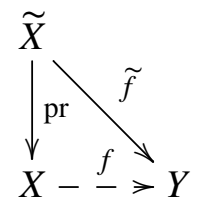

commute (wherever $f \circ \mathrm{pr}$ is defined). Then, one can show that

$$
f^{*} \alpha=\operatorname{pr}_{*}(\tilde{f})^{*} \alpha
$$

see, for example, [R, Lemma 3.1]. Notice that for any rational map $f: X \rightarrow-\rightarrow$, the space $\widetilde{X}$ and maps pr and $\widetilde{f}$ always exist: for example, $\widetilde{X}$ can be obtained as a desingularization of $\Gamma_{f}$, with the maps pr and $\tilde{f}$ corresponding to the lifts of $\left.\pi_{1}\right|_{\Gamma_{f}}$ and $\left.\pi_{2}\right|_{\Gamma_{f}}$.

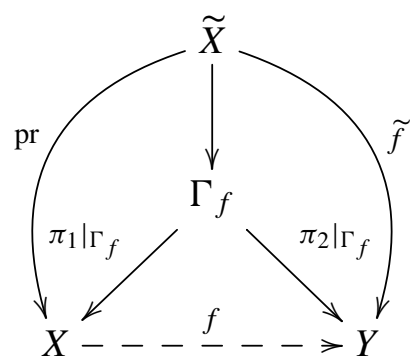

For any Kähler manifold $X$, there is a natural isomorphism

$$
\bigoplus_{p+q=i} H^{p, q}(X ; \mathbb{C}) \rightarrow H^{i}(X ; \mathbb{C}),
$$

where the former are the Dolbeault cohomology groups and the latter is the singular cohomology. This induces a splitting on the singular cohomology of $X$ into bidegrees. To see that (2) preserves this splitting, observe that (4) can be applied to any $\bar{\partial}$-closed $(p, q)$-form $\beta$, with $(\widetilde{f})^{*}$ interpreted as the pullback on smooth forms and $\mathrm{pr}_{*}$ interpreted 
as the proper pushforward on currents of degree $(p, q)$. As both of these operations induce a well-defined map on cohomology, we see that the $f^{*}[\beta]=\operatorname{pr}_{*}(\tilde{f})^{*}[\beta]=\left[\operatorname{pr}_{*}(\tilde{f})^{*} \beta\right] \in$ $H^{p, q}(X ; \mathbb{C})$. In particular, the pullback defined by $(2)$ can be used in the definition of the dynamical degree $\lambda_{k}$ for any $1 \leqslant k \leqslant N$.

We note that many authors define the pullback on cohomology using forms and currents as above, rather than the singular cohomology approach we have used. For more discussion of the latter approach, see [R].

We will use the following criterion for functoriality of compositions, which is proved in [DS3, Am, R].

Proposition 2.1. Let $X, Y$, and $Z$ be projective manifolds of equal dimension, and let $f: X \rightarrow Y$ and $g: Y \rightarrow Z$ be dominant rational maps. Suppose that there exist a projective manifold $\widetilde{X}$ and holomorphic maps pr and $\widetilde{f}$ making the diagram

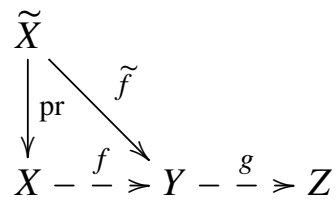

commute (wherever $f \circ \mathrm{pr}$ is defined), with the property that $\tilde{f}^{-1}(x)$ is a finite set for every $y \in Y$. Then $(g \circ f)^{*}=f^{*} \circ g^{*}$ on all cohomology groups.

Remark 2.2. Note that it follows from the criterion of Bedford and Kim [BK2, Theorem 1.1] that if $f_{X \backslash I_{f}}: X \backslash I_{f} \rightarrow X$ is finite then $f$ is 1-stable. This is not sufficient for $k$-stability when $k>1$, as shown in [R, Proposition 6.1]. This is why we use the stronger sufficient condition in Proposition 2.1.

The following lemma (see, for example, [Fu, Lemma 19.1.2]) will be helpful when using (4) to compute pullbacks.

Lemma 2.3. Suppose that $f: X \rightarrow Y$ is a proper holomorphic map between projective manifolds. For any irreducible subvariety $V \subseteq X$ :

(i) if $\operatorname{dim}(f(V))=\operatorname{dim}(V)$, then $f_{*}([V])=\operatorname{deg}_{\text {top }}\left(\left.f\right|_{V}\right)[f(V)]$, where $\operatorname{deg}_{\text {top }}\left(\left.f\right|_{V}\right)$ is the number of preimages under $\left.f\right|_{V}$ of a generic point from $f(V)$.

(ii) Otherwise, $f_{*}([V])=0$.

3. Moduli space

Let $P=\left\{p_{1}, \ldots, p_{n}\right\}$ be a finite set consisting of at least three points. The moduli space of genus 0 curves marked by $P$ is by definition

$$
\mathcal{M}_{P}:=\left\{\varphi: P \hookrightarrow \mathbb{P}^{1} \text { up to postcomposition by Möbius transformations }\right\} .
$$

3.1. Projective space. Every element of $\mathcal{M}_{P}$ has a representative $\varphi: P \hookrightarrow \mathbb{P}^{1}$ so that

$$
\varphi\left(p_{1}\right)=0 \quad \text { and } \varphi\left(p_{2}\right)=\infty,
$$

and the point $[\varphi] \in \mathcal{M}_{P}$ is determined by the $(n-2)$-tuple

$$
\left(z_{1}, \ldots, z_{n-2}\right) \in \mathbb{C}^{n-2} \quad \text { where } z_{i}:=\varphi\left(p_{i+2}\right), \text { for } 1 \leqslant i \leqslant n-2,
$$


up to scaling by a non-zero complex number. In other words, the point $[\varphi] \in \mathcal{M}_{P}$ is uniquely determined by $\left[z_{1}: \cdots: z_{N+1}\right] \in \mathbb{P}^{N}$, where $N:=n-3$. There are some immediate constraints on the complex numbers $z_{i}$ in order to ensure that $\varphi: P \hookrightarrow \mathbb{P}^{1}$ is injective. Indeed, $\mathcal{M}_{P}$ is isomorphic to the complement of $(n-1)(n-2) / 2$ hyperplanes in $\mathbb{P}^{N}$. We state this in the following proposition.

Proposition 3.1. Define $z_{0}:=0$. The moduli space $\mathcal{M}_{P}$ is isomorphic to $\mathbb{P}^{N} \backslash \Delta$ where $\Delta$ is the following collection of hyperplanes:

$$
\Delta:=\left\{z_{i}=z_{j} \mid 0 \leqslant i<j \leqslant N+1\right\} .
$$

In particular, $\mathcal{M}_{P}$ is a complex manifold of dimension $N$.

Proof. This follows immediately from the normalization above.

The following fact is straightforward, but we state it explicitly as is will be used in subsequent sections.

Proposition 3.2. Let $Q=\left\{q_{1}, \ldots, q_{n}\right\}$, and let $\iota: P \rightarrow Q$ be a bijection. Then $\iota$ induces an isomorphism

$$
\iota^{*}: \mathcal{M}_{Q} \rightarrow \mathcal{M}_{P}
$$

Proof. Let $m \in \mathcal{M}_{Q}$, and let $\varphi: Q \hookrightarrow \mathbb{P}^{1}$ be a representative of $m$. Then $\iota^{*}(m) \in \mathcal{M}_{P}$ is represented by $\varphi \circ \iota: P \hookrightarrow \mathbb{P}^{1}$.

The moduli space $\mathcal{M}_{P}$ is not compact.

3.2. The Deligne-Mumford compactification. A stable curve of genus 0 marked by $P$ is an injection $\varphi: P \hookrightarrow C$ where $C$ is a connected algebraic curve whose singularities are ordinary double points (called nodes), such that:

(1) each irreducible component is isomorphic to $\mathbb{P}^{1}$;

(2) the graph, $G_{C}$, whose vertices are the irreducible components and whose edges connect components intersecting at a node, is a tree;

(3) for all $p \in P, \varphi(p)$ is a smooth point of $C$; and

(4) the number of marked points plus nodes on each irreducible component of $C$ is at least 3.

The marked stable curves $\varphi_{1}: P \hookrightarrow C_{1}$ and $\varphi_{2}: P \hookrightarrow C_{2}$ are isomorphic if there is an isomorphism $\mu: C_{1} \rightarrow C_{2}$ such that $\varphi_{2}=\mu \circ \varphi_{1}$. The set of stable curves of genus 0 marked by $P$ modulo isomorphism can be given the structure of a smooth projective variety $[\mathbf{K n}, \mathbf{K M}]$, called the Deligne-Mumford compactification, and denoted by $\overline{\mathcal{M}}_{P}$. The moduli space $\mathcal{M}_{P}$ is an open Zariski dense subset of $\overline{\mathcal{M}}_{P}$. In this subsection we will state some of the well-known properties of $\overline{\mathcal{M}}_{P}$.

Let $P=\left\{p_{1}, \ldots, p_{n}\right\}$ be a set with at least three elements. The compactification divisor of $\mathcal{M}_{P}$ in $\overline{\mathcal{M}}_{P}$ is the set of all (isomorphism classes) of marked stable curves with at least one node. Generic points of $\overline{\mathcal{M}}_{P} \backslash \mathcal{M}_{P}$ consist of the (isomorphism classes) of marked stable curves $\varphi: P \hookrightarrow C$ with at exactly one node. For each such generic boundary point, taking $\varphi^{-1}$ of the connected components of $C \backslash\{$ node $\}$ induces a partition of $P$ into two 
sets $S \cup S^{c}$, where $S^{c}:=P \backslash S$. The set of generic boundary components inducing a given partition of $P$ is an irreducible quasiprojective variety and its closure in $\overline{\mathcal{M}}_{P}$ is an algebraic hypersurface denoted $\mathcal{D}^{S} \equiv \mathcal{D}^{S^{c}}$, and it is a boundary divisor of $\overline{\mathcal{M}}_{P}$.

If $|S|=n_{1}$ and $\left|S^{C}\right|=n_{2}$ then there is an isomorphism

$$
\mathcal{D}^{S} \approx \overline{\mathcal{M}}_{0, n_{1}+1} \times \overline{\mathcal{M}}_{0, n_{2}+1}
$$

The $n_{1}+1$ points in the first factor consist of the $n_{1}$ points of $S$ together with the node, and similarly for the second factor.

Any $k$ distinct boundary divisors $\mathcal{D}^{S_{1}}, \ldots, \mathcal{D}^{S_{k}}$ intersect transversally, and if this intersection is non-empty, the result is an irreducible codimension $k$ boundary stratum. This corresponds to the set of marked stable curves which induces a stable partition of $P$ into $k+1$ blocks. There is an analogous description in terms of trees.

Marked stable trees. Let $\varphi: P \hookrightarrow C$ be a marked stable curve of genus 0 . As mentioned above in property (2) of such a curve, there is a graph $G_{C}$ associated to $\varphi: P \hookrightarrow C$, which is a tree. Let $V_{C}$ be the set of vertices of $G_{C}$; the marking $\varphi: P \hookrightarrow C$ induces a map $\varphi_{*}: P \rightarrow V_{C}$, sending $p \in P$ to the vertex corresponding to the irreducible component which contains $\varphi(p)$. We will call

$$
T_{\varphi: P \hookrightarrow C}:=\left(G_{C}, \varphi_{*}: P \rightarrow V_{C}\right)
$$

the marked stable tree associated to the marked stable curve $\varphi: P \hookrightarrow C$. More generally, any graph $G$ which is a tree, together with a map $\psi: P \rightarrow V_{G}$, will be a marked stable tree if for all $v \in V_{G}$,

$$
\operatorname{degree}(v)+\left|\psi^{-1}(v)\right| \geqslant 3 \text {. }
$$

Given two generic points $\varphi: P \hookrightarrow C$ and $\varphi^{\prime}: P \hookrightarrow C^{\prime}$ of the (non-empty) intersection $\mathcal{D}^{S_{1}} \cap \cdots \cap \mathcal{D}^{S_{k}}$, the trees $T_{\varphi: P \hookrightarrow C}$ and $T_{\varphi^{\prime}: P \hookrightarrow C^{\prime}}$ are isomorphic in the following sense: there is a graph isomorphism $\beta: G_{C} \rightarrow G_{C^{\prime}}$ so that $\varphi_{*}^{\prime}=\beta \circ \varphi_{*}$. The stratum $\mathcal{D}^{S_{1}} \cap \cdots \cap \mathcal{D}^{S_{k}}$ can be labeled by the isomorphism class of $T_{\varphi: P \hookrightarrow C}$.

It is well known that there is a bijection between the following sets:

$$
\text { \{codimension } \left.k \text { boundary strata in } \overline{\mathcal{M}}_{P}\right\} \longleftrightarrow
$$

\{isomorphism classes of marked stable trees with $k+1$ vertices\}.

LEMMA 3.3. Let $Z$ be a boundary stratum of codimension $k$ in $\overline{\mathcal{M}}_{P}$. There is a unique set $\left\{\mathcal{D}^{S_{1}}, \ldots, \mathcal{D}^{S_{k}}\right\}$ of boundary divisors so that $Z=\mathcal{D}^{S_{1}} \cap \cdots \cap \mathcal{D}^{S_{k}}$.

Proof. This result follows immediately from the remarks above.

3.3. Keel's theorem. In [Ke], Keel exhibits generators and relations for the cohomology ring of $\overline{\mathcal{M}}_{P}$. Let $\left[\mathcal{D}^{S}\right]$ denote the fundamental cohomology class of the boundary divisor $\mathcal{D}^{S}$.

THEOREM 3.4. [Ke] The cohomology ring $H^{*}\left(\overline{\mathcal{M}}_{P} ; \mathbb{C}\right)$ is the ring

$$
\mathbb{Z}\left[\left[\mathcal{D}^{S}\right]: S \subseteq P,|S|,\left|S^{c}\right| \geqslant 2\right]
$$


modulo the following relations:

(1) $\left[\mathcal{D}^{S}\right]=\left[\mathcal{D}^{S^{c}}\right]$.

(2) For any four distinct $p_{i}, p_{j}, p_{k}, p_{l} \in P$,

$$
\sum_{\substack{p_{i}, p_{j} \in S \\ p_{k}, p_{l} \in S^{c}}}\left[\mathcal{D}^{S}\right]=\sum_{\substack{p_{i}, p_{k} \in S \\ p_{j}, p_{l} \in S^{c}}}\left[\mathcal{D}^{S}\right]=\sum_{\substack{p_{i}, p_{l} \in S \\ p_{j}, p_{k} \in S^{c}}}\left[\mathcal{D}^{S}\right] .
$$

(3) $\left[\mathcal{D}^{S}\right] \smile\left[\mathcal{D}^{T}\right]=0$ unless one of the following holds:

$$
S \subseteq T, \quad T \subseteq S, \quad S \subseteq T^{c}, \quad T^{c} \subseteq S .
$$

Implicit in Keel's theorem is the assertion that the codimension $k$ boundary strata are complete intersections.

COROLlaRY 3.5. We have

$$
\left[\mathcal{D}^{S_{1}} \cap \cdots \cap \mathcal{D}^{S_{k}}\right]=\left[\mathcal{D}^{S_{1}}\right] \smile \cdots \smile\left[\mathcal{D}^{S_{k}}\right] .
$$

We now construct Kapranov's space $X^{N}$ which is isomorphic to $\overline{\mathcal{M}}_{P}$.

3.4. Kapranov's theorem. We may choose coordinates and identify $\mathcal{M}_{P}$ with $\mathbb{P}^{N} \backslash \Delta$ as stated in Proposition 3.1. In this concrete setting, there is a description of $\overline{\mathcal{M}}_{P}$ as a sequential blow-up of $\mathbb{P}^{N}$ due to Kapranov [Ka].

Normalize to identify $\mathcal{M}_{P}$ with $\mathbb{P}^{N} \backslash \Delta$ as in Proposition 3.1, and consider the following subsets of $\mathbb{P}^{N}$. Let

$$
A^{0}:=\{[1: 0: \cdots: 0],[0: 1: 0: \cdots: 0], \ldots,[0: \cdots: 0: 1],[1: 1: \cdots: 1]\}
$$

and, for $1 \leqslant i \leqslant N-2$, let $A^{i}$ be the set of all $\left(\begin{array}{c}N+2 \\ i+1\end{array}\right)$ projective linear subspaces of dimension $i$ in $\mathbb{P}^{N}$, which are spanned by collections of $i+1$ distinct points in $A^{0}$. Let $X_{0}:=\mathbb{P}^{N}$, and for each $0 \leqslant i \leqslant N-2$ define $\alpha^{i}: X_{i+1} \rightarrow X_{i}$ to be the blow-up of $X_{i}$ along the proper transform $\widetilde{A}^{i}$ of $A^{i}$ under $\alpha^{0} \circ \cdots \circ \alpha^{i-1}$.

THEOREM 3.6. [Ka] Let $P=\left\{p_{1}, \ldots, p_{n}\right\}$ contain at least three points. Normalize to identify $\mathcal{M}_{P}$ with $\mathbb{P}^{N} \backslash \Delta$ where $N=n-3$ as in Proposition 3.1. Then the DeligneMumford compactification $\overline{\mathcal{M}}_{P}$ is isomorphic to the space $X^{N}:=X_{N-1}$ constructed above.

The proof of Kapranov's theorem is slightly subtle, establishing the isomorphism using the space of Veronese curves. For a different perspective, closer to that of the present paper, we refer the reader to the paper of Harvey and Lloyd-Philipps [HL].

Remark 3.7. Via the isomorphism $\overline{\mathcal{M}}_{P} \approx X^{N}$ from Theorem 3.6, we will use Theorem 3.4 to find appropriate bases for the cohomology groups $H^{k, k}\left(X^{N} ; \mathbb{C}\right)$ in $\S 6$. To this end, we adopt the following notation. Let $\mathcal{D}^{S} \subseteq \overline{\mathcal{M}}_{P}$ be a boundary divisor. We will use the notation $D^{S} \subseteq X^{N}$ to denote the image of $\mathcal{D}^{S}$ under the explicit isomorphism $\overline{\mathcal{M}}_{P} \approx X^{N}$ from Theorem 3.6.

For $|P|=3, \mathcal{M}_{P}=\overline{\mathcal{M}}_{P}$ is a point. For $|P|=4, \mathcal{M}_{P}$ is isomorphic to $\mathbb{P}^{1} \backslash\{0,1, \infty\}$, and $\overline{\mathcal{M}}_{P}$ is isomorphic to $\mathbb{P}^{1}$. 

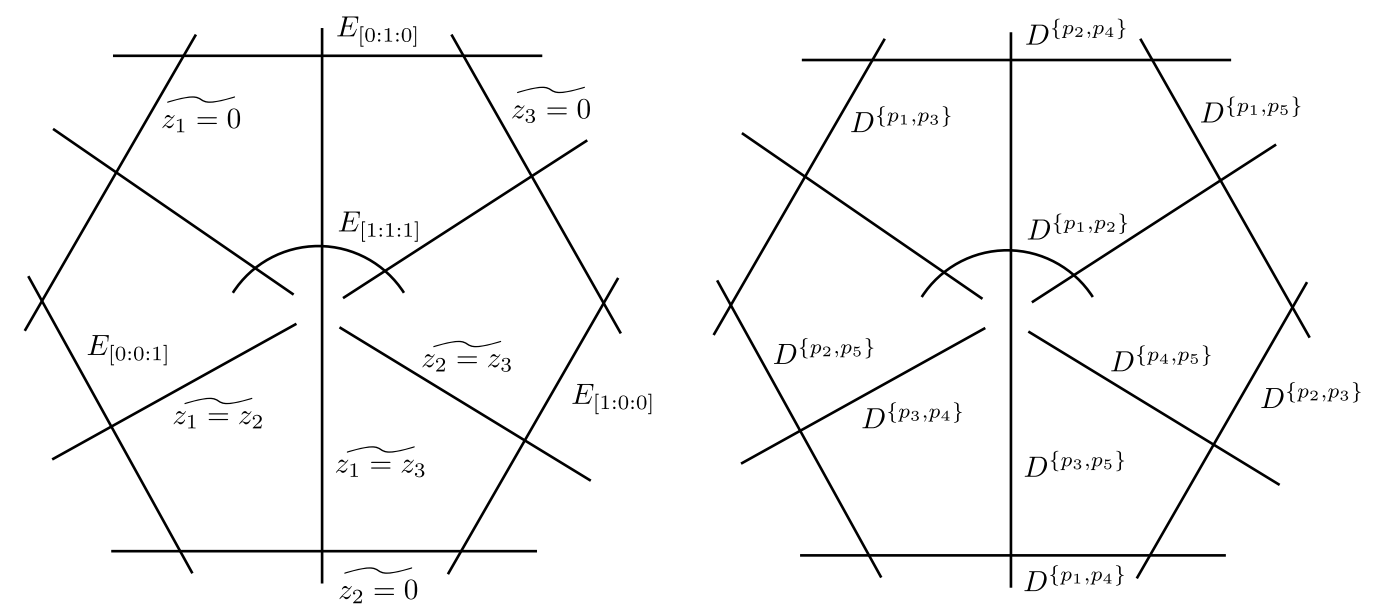

FIGURE 1. Depiction of $X^{2}$. Left: boundary divisors are labeled as proper transforms of lines in $\mathbb{P}^{2}$ and exceptional divisors. Right: boundary divisors are labeled according to Remark 3.7.

Example 3.8. Let $P=\left\{p_{1}, p_{2}, p_{3}, p_{4}, p_{5}\right\}$. Following Proposition 3.1, $\mathcal{M}_{P}$ is isomorphic to $\mathbb{P}^{2} \backslash \Delta$, where

$$
\Delta=\left\{z_{1}=0, z_{2}=0, z_{3}=0, z_{1}=z_{2}, z_{2}=z_{3}, z_{1}=z_{3}\right\} .
$$

The space $\overline{\mathcal{M}}_{P}$ is isomorphic to $X^{2}$, which is equal to $\mathbb{P}^{2}$ blown up at the four points comprising $A^{0}$ :

$$
\{[1: 0: 0],[0: 1: 0],[0: 0: 1],[1: 1: 1]\} .
$$

There are 10 boundary divisors in $X^{2}$ : the proper transforms of the six lines comprising $\Delta$, plus the four exceptional divisors. The ten boundary divisors correspond to the $\left(\begin{array}{l}5 \\ 2\end{array}\right)$ stable partitions of $P$ into two blocks. The space $X^{2}$ is depicted in Figure 1.

Example 3.9. If $|P|=6$, then $\overline{\mathcal{M}}_{P}$ is isomorphic to $X^{3}$, the sequential blow-up of $\mathbb{P}^{3}$ where

$$
A^{0}=\{[0: 0: 0: 1],[0: 0: 1: 0],[0: 1: 0: 0],[1: 0: 0: 0],[1: 1: 1: 1]\}
$$

and $A^{1}$ is the set of $10=\left(\begin{array}{l}5 \\ 2\end{array}\right)$ lines spanned by pairs of points in $A^{0}$. A depiction of $X^{3}$ is shown in Figure 2.

Example 3.10. If $|P|=7$, then $\overline{\mathcal{M}}_{P}$ is isomorphic to $X^{4}$, the sequential blow-up of $\mathbb{P}^{4}$ where

$$
\begin{aligned}
A^{0}= & \{[0: 0: 0: 0: 1],[0: 0: 0: 1: 0],[0: 0: 1: 0: 0],[0: 1: 0: 0: 0],[1: 0: 0: 0: 0], \\
& {[1: 1: 1: 1: 1]\}, }
\end{aligned}
$$

$A^{1}$ is the set of 15 lines spanned by pairs of points in $A^{0}$, and $A^{2}$ is the set of 20 planes spanned by triples of points in $A^{0}$. Let $\left[x_{1}: x_{2}: x_{3}: x_{4}: x_{5}\right] \in \mathbb{P}^{4}$, let $L$ be the element of $A^{2}$ spanned by

$$
\{[0: 0: 0: 0: 1],[0: 0: 0: 1: 0],[0: 0: 1: 0: 0]\}
$$




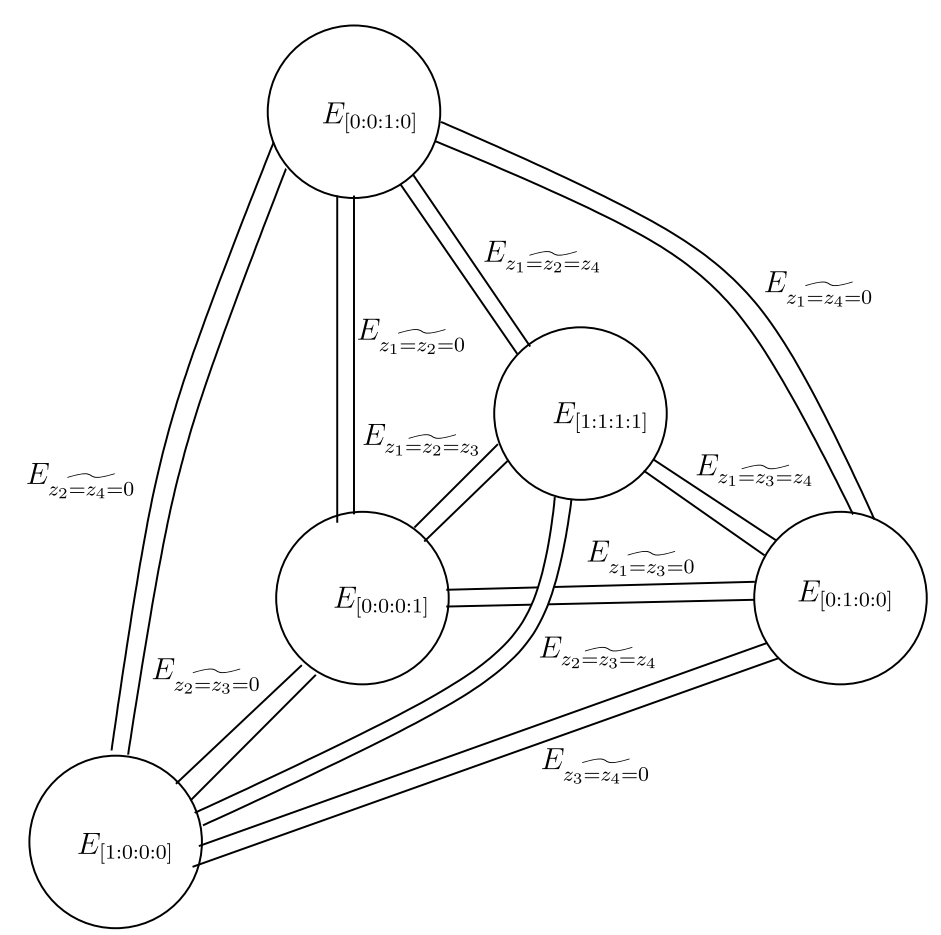

FIGURE 2. Depiction of $X^{3}$ with all boundary divisors corresponding to exceptional divisors over $A^{0}$ and over proper transforms of lines from $A^{1}$ labeled. (The remaining 10 boundary divisors corresponding to hyperplanes in $\mathbb{P}^{3}$ are not labeled.)

that is, the locus in $\mathbb{P}^{4}$ given by $x_{1}=x_{2}=0$, and let $M$ be the element in $A^{2}$ spanned by

$$
\{[0: 1: 0: 0: 0],[1: 0: 0: 0: 0],[1: 1: 1: 1: 1]\}
$$

that is, the locus in $\mathbb{P}^{4}$ given by $x_{3}=x_{4}=x_{5}$. Note that $L$ and $M$ intersect at the point $[0: 0: 1: 1: 1]$ which is not in $A^{0} \cup A^{1}$, so a priori, the order of the blow-ups in the construction above might matter in constructing the space $X^{4}$. However, this is not the case since $L$ and $M$ intersect transversally. Indeed, this phenomenon occurs in the general setting for $|P|$ arbitrary, but these intersections are always transverse and are therefore irrelevant in the blow-up construction (see Lemmas 5.3 and 5.4).

3.5. Automorphisms of $X^{N}$. The automorphism group of $X^{N}$ is clearly isomorphic to the automorphism group of $\overline{\mathcal{M}}_{P}$. We will study automorphisms of $\overline{\mathcal{M}}_{P}$ that extend automorphisms of $\mathcal{M}_{P}$. If $|P|=4$, then the automorphisms of $\overline{\mathcal{M}}_{P} \approx \mathbb{P}^{1}$ that extend the automorphisms of $\mathcal{M}_{P}$ consist of the Möbius transformations that map the set of three points comprising the boundary of $\mathcal{M}_{P}$ in $\overline{\mathcal{M}}_{P}$ to itself; that is, $\operatorname{Aut}\left(\mathcal{M}_{P}\right)$ is isomorphic to the permutation group on three letters. If $|P|>4$, then $\operatorname{Aut}\left(\mathcal{M}_{P}\right)$ is isomorphic to $S_{P}$, the group of permutations of elements of the set $P$ (see [T], and compare with Proposition 3.2).

Proposition 3.11. Suppose that $|P|>4$, and let $\rho \in S_{P}$. Then the automorphism $g_{\rho}: \mathcal{M}_{P} \rightarrow \mathcal{M}_{P}$ extends to an automorphism $g_{\rho}: \overline{\mathcal{M}}_{P} \rightarrow \overline{\mathcal{M}}_{P}$. 
Proof. Let $P=\left\{p_{1}, \ldots, p_{n}\right\}$, and let $\rho \in S_{P}$. The permutation $\rho$ relabels the points in $P$, which effectively just changes coordinates on $\overline{\mathcal{M}}_{P}$. This is evident using Kapranov's theorem from $\$ 3.4$. Indeed, in the construction of $X^{N}$, we began with a choice of normalization: we identified $\mathcal{M}_{P}$ with a $\mathbb{P}^{N} \backslash \Delta$ by choosing a representative $\varphi: P \hookrightarrow \mathbb{P}^{1}$ so that $\varphi\left(p_{1}\right)=0$, and $\varphi\left(p_{2}\right)=\infty$, and setting $z_{i}:=\varphi\left(p_{i+2}\right)$ for $1 \leqslant i \leqslant n-2$, we identified the point $[\varphi] \in \mathcal{M}_{P}$ with the point $\left[z_{1}: \cdots: z_{N+1}\right] \in \mathbb{P}^{N}$. To build $X^{N}$, we performed the appropriate sequential blow-up of this copy of $\mathbb{P}^{N}$.

Carrying out the same construction, but taking the permutation into account, we normalize so that for the representative $\varphi: P \hookrightarrow \mathbb{P}^{1}$,

$$
\varphi\left(p_{\rho^{-1}(1)}\right)=0 \quad \text { and } \quad \varphi\left(p_{\rho^{-1}(2)}\right)=\infty,
$$

and by setting $z_{i}:=\varphi\left(p_{\rho^{-1}(i)}\right)$, for $1 \leqslant i \leqslant n-2$, we identify the point $[\varphi] \in \mathcal{M}_{P}$ with the point $\left[z_{1}: \cdots: z_{M+1}\right] \in \mathbb{P}^{M}$, where $M:=n-3$. Build a space $Y^{M}$ which is the sequential blow-up of $\mathbb{P}^{M}$ as prescribed in $\$ 3.4$ (we have changed notation so as not to confuse the two constructions of the 'same' space). The spaces $X^{N}$ and $Y^{M}$ are clearly isomorphic, and we see that $\rho$ induces an automorphism $g_{\rho}: \overline{\mathcal{M}}_{P} \rightarrow \overline{\mathcal{M}}_{P}$ which extends $g_{\rho}: \mathcal{M}_{P} \rightarrow \mathcal{M}_{P}$.

\section{The maps $f_{\rho}: X^{N} \rightarrow X^{N}$}

As previously mentioned, the maps $f_{\rho}: X^{N} \rightarrow X^{N}$ will be a composition of two maps: an automorphism $g_{\rho}: X^{N} \rightarrow X^{N}$ and a map $s: X^{N} \rightarrow X^{N}$, which we now define.

Let $P=\left\{p_{1}, p_{2}, \ldots, p_{n}\right\}$, normalize to identify $\mathcal{M}_{P}$ with $\mathbb{P}^{N} \backslash \Delta$ as in Proposition 3.1, and, via Kapranov's construction (Theorem 3.6), build the space $X^{N}$ as a sequential blowup of $\mathbb{P}^{N}$. Consider the squaring map $s_{0}: \mathbb{P}^{N} \rightarrow \mathbb{P}^{N}$ given by

$$
s_{0}:\left[z_{1}: \cdots: z_{N+1}\right] \mapsto\left[z_{1}^{2}: \cdots z_{N+1}^{2}\right],
$$

which is clearly holomorphic. Note that the critical locus of $s_{0}$ consists precisely of the union of hyperplanes

$$
\operatorname{Crit}\left(s_{0}\right)=\bigcup_{i=1}^{N+1}\left\{z_{i}=0\right\} .
$$

Moreover, every component of $\Delta$ is mapped to itself by $s_{0}$.

The map $s: X^{N} \rightarrow X^{N}$ is simply the lift of $s_{0}: \mathbb{P}^{N} \rightarrow \mathbb{P}^{N}$ under the map $\mathcal{A}:=\alpha_{0} \circ \cdots \circ \alpha_{N-2}: X_{N-1} \rightarrow X_{0}$ where $X^{N}:=X_{N-1}$ and $X_{0}:=\mathbb{P}^{N}$ in the Kapranov construction (see Theorem 3.6). The map $s: X^{N} \rightarrow X^{N}$ is not holomorphic (unless $N=1$, or equivalently, $|P|=4$ ); indeed, there are points of indeterminacy arising from extra preimages of varieties that were previously blown up. For example, consider $P=$ $\left\{p_{1}, \ldots, p_{5}\right\}$ (as in Example 3.8). The space $X^{2}$ is $\mathbb{P}^{2}$ blown up at $[0: 0: 1],[0: 1$ : $0]$, [1:0:0], and $[1: 1: 1]$. Let $\zeta \in\left(s_{0}^{-1}([1: 1: 1]) \backslash\{[1: 1: 1]\}\right)$. Then $s: X^{2} \rightarrow-X^{2}$ has a point of indeterminacy at $\alpha_{0}^{-1}(\zeta)$. In fact, for any $N>1$ the indeterminacy set for $s: X^{N} \rightarrow X^{N}$ has dimension $N-2$. Notice that $\left\{z_{1}=z_{2}=-z_{3}\right\} \in s_{0}^{-1}\left(\left\{z_{1}=z_{2}=z_{3}\right\}\right)$, with $\left\{z_{1}=z_{2}=z_{3}\right\} \in A^{N-2}$. Since $\left\{z_{1}=z_{2}=-z_{3}\right\}$ is not a center of blow-up, its proper transform under $\mathcal{A}$ is in the indeterminacy locus $I_{S}$. 
By Proposition 3.11, every permutation $\rho \in S_{P}$ induces an automorphism $g_{\rho}: \overline{\mathcal{M}}_{P} \rightarrow \overline{\mathcal{M}}_{P}$, which maps the compactification divisor of $\mathcal{M}_{P}$ to itself (since $g_{\rho}$ extends an automorphism of $\left.\mathcal{M}_{P}\right)$. We abuse notation and denote the corresponding automorphism of $X^{N} \rightarrow X^{N}$ by $g_{\rho}: X^{N} \rightarrow X^{N}$.

For any $\rho \in S_{P}$, define the map $f_{\rho}:=g_{\rho} \circ s: X^{N} \rightarrow X^{N}$. This map also has indeterminacy locus of dimension $N-2$, since $I_{g_{\rho} \circ s}=I_{s}$. We now prove that the maps $f_{\rho}: X^{N} \rightarrow X^{N}$ are algebraically stable.

\section{Algebraic stability}

The goal of this section is to prove the following proposition, which will imply Theorem 1.1 and will be used to compute the linear maps $\left(f_{\rho}\right)^{*}: H^{k, k}\left(X^{N} ; \mathbb{C}\right) \rightarrow$ $H^{k, k}\left(X^{N} ; \mathbb{C}\right)$.

Proposition 5.1. For any $N \geqslant 1$ there is an $N$-dimensional projective manifold $Y^{N}$ and holomorphic maps pr: $Y^{N} \rightarrow X^{N}$ and $\widetilde{s}: Y^{N} \rightarrow X^{N}$ that make the diagram

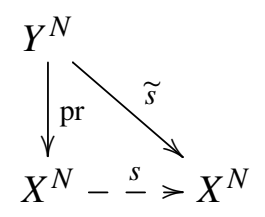

commute (wherever $s$ o $\operatorname{pr}$ is defined), with $\widetilde{s}^{-1}(x)$ a finite set for every $x \in X^{N}$.

Proof of Theorem 1.1, supposing Proposition 5.1. Using the factorization $f_{\rho}=g_{\rho} \circ s$ with $g_{\rho}$ an automorphism of $X^{N}$, we obtain the diagram

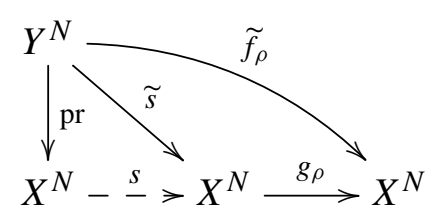

Since $\widetilde{s}$ has finite fibers, so does $\tilde{f}_{\rho}:=g_{\rho} \circ \widetilde{s}$. It follows from Proposition 2.1 that for any rational map $h: X^{N} \rightarrow X^{N}$ we have $\left(h \circ f_{\rho}\right)^{*}=f_{\rho}^{*} \circ h^{*}$ on all cohomology groups. By induction it follows that for any word $f_{\rho_{k}} \circ \cdots \circ f_{\rho_{1}}$,

$$
\left(f_{\rho_{k}} \circ \cdots \circ f_{\rho_{1}}\right)^{*}=f_{\rho_{1}}^{*} \circ \cdots \circ f_{\rho_{k}}^{*}
$$

on $H^{k, k}\left(X^{N} ; \mathbb{C}\right)$ for $1 \leqslant k \leqslant N$. This implies that the semi-group $\mathcal{F}^{N}$ acts functorially on all of the cohomology groups of $X^{N}$.

In order to prove Proposition 5.1, we will use the universal property of blow-ups, following the treatment in $[\mathbf{E H}, \mathbf{G W}$. Let $X$ be any scheme and $Y \subseteq X$ a subscheme. Recall that $Y$ is a Cartier subscheme if it is locally the zero locus of a single regular function.

Universal property. Let $X$ be a scheme and let $Y$ be a closed subscheme. The blow-up of $X$ along $Y$ is a scheme $\widetilde{X} \equiv \operatorname{BL}_{Y}(X)$ and a morphism $\pi: \widetilde{X} \rightarrow X$ such that $\pi^{-1}(Y)$ is a Cartier subscheme and which is universal with respect to this property: if $\pi^{\prime}: \tilde{X}^{\prime} \rightarrow X$ is 
any morphism such that $\left(\pi^{\prime}\right)^{-1}(Y)$ is a Cartier subscheme, then there is a unique morphism $g: \tilde{X}^{\prime} \rightarrow \tilde{X}$ such that $\pi^{\prime}=\pi \circ g$.

Recall that the Cartier subscheme $E=\pi^{-1}(Y)$ is called the exceptional divisor of the blow-up and $Y$ is called the center of the blow-up.

There is an immediate corollary of the definition; see, for example, [GW, Proposition 13.91].

COROllary 5.2. Let $X$ be a scheme, let $Y$ be a closed subscheme, and let $\pi: \mathrm{BL}_{Y}(X) \rightarrow X$ be the blow-up of $X$ along $Y$. Let $f: X^{\prime} \rightarrow X$ be any morphism of schemes. Then there exists a unique morphism $\mathrm{BL}_{Y}(f): \operatorname{BL}_{f^{-1}(Y)}\left(X^{\prime}\right) \rightarrow \operatorname{BL}_{Y}(X)$ making the following diagram commute:

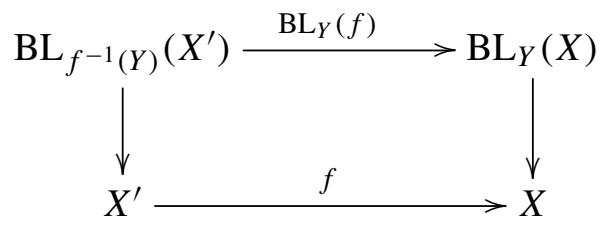

In our context, $X$ will be a projective manifold and $Y \subseteq X$ will be a projective submanifold.

The following is well known, but we include a proof for completeness.

LEMMA 5.3. Suppose that $X$ is a projective manifold and $Y, Z \subseteq X$ are projective submanifolds that intersect transversally (i.e. $T_{x} Y+T_{x} Z=T_{x} X$ at any $x \in Y \cap Z$ ). Then:

(1) if $\pi: \mathrm{BL}_{Y}(X) \rightarrow X$ is the blow-up, the proper transform $\widetilde{Z}=\overline{\pi^{-1}(Z \backslash Y)}$ and total transform $\pi^{-1}(Z)$ coincide;

$\mathrm{BL}_{\widetilde{Z}}\left(\mathrm{BL}_{Y}(X)\right) \cong \mathrm{BL}_{\widetilde{Y}}\left(\mathrm{BL}_{Z}(X)\right)$.

Proof. Since the blow-up along a submanifold is a local construction, it suffices to check this statement when $X=\mathbb{C}^{N}, Y=\operatorname{span}\left(e_{1}, \ldots, e_{k}\right)$, and $Z=\operatorname{span}\left(e_{l}, \ldots, e_{N}\right)$, where $e_{1}, \ldots, e_{N}$ are the standard basis vectors in $\mathbb{C}^{N}$. Since $Y$ and $Z$ are assumed transverse, $l \leqslant k+1$. We have

$$
\begin{aligned}
\operatorname{BL}_{Y}(X)=\{ & \left(x_{1}, \ldots, x_{N}\right) \times\left[m_{k+1}: \ldots: m_{N}\right] \in \mathbb{C}^{N} \\
& \left.\times \mathbb{P}^{N-k-1} \mid\left(m_{k+1}, \ldots, m_{N}\right) \sim\left(x_{k+1}, \ldots, x_{N}\right)\right\},
\end{aligned}
$$

where $\mathbf{u} \sim \mathbf{v}$ means that one vector is a scalar multiple of the other. Notice that

$$
\begin{aligned}
\widetilde{Z}=\{ & \left(0, \ldots, 0, x_{l}, \ldots, x_{N}\right) \times\left[m_{k+1}: \ldots: m_{N}\right] \in \mathbb{C}^{N} \\
& \left.\times \mathbb{P}^{N-k-1} \mid\left(m_{k+1}, \ldots, m_{N}\right) \sim\left(x_{k+1}, \ldots, x_{N}\right)\right\},
\end{aligned}
$$

which coincides with $\pi^{-1}(Z)$, proving (1).

If we blow up $\widetilde{Z}$, we find that

$$
\begin{aligned}
\operatorname{BL}_{\widetilde{Z}}\left(\operatorname{BL}_{Y}(X)\right)=\{ & \left(x_{1}, \ldots, x_{N}\right) \times\left[m_{k+1}: \ldots: m_{N}\right] \times\left[n_{1}: \ldots: n_{l-1}\right] \in \mathbb{C}^{N} \\
& \times \mathbb{P}^{N-k-1} \times \mathbb{P}^{l-2} \mid\left(m_{k+1}, \ldots, m_{N}\right) \sim\left(x_{k+1}, \ldots, x_{N}\right) \text { and } \\
& \left.\left(n_{1}, \ldots, n_{l-1}\right) \sim\left(x_{1}, \ldots, x_{l-1}\right)\right\} .
\end{aligned}
$$

This is clearly isomorphic to the result we would obtain if we had first blown up $Z$ and then blown up $\tilde{Y}$, proving (2). 
We will break the proof of Proposition 5.1 into Lemmas 5.6 and 5.8, below. In order to keep notation as simple as possible, we will usually drop the dimension $N$ from the notation, writing $X \equiv X^{N}$ and $Y \equiv Y^{N}$.

In order to construct $Y$, we first recall the construction of $X$. Recall that

$$
A_{0}=\{[1: 0: \cdots: 0],[0: 1: 0: \cdots: 0], \ldots,[0: \cdots: 0: 1],[1: 1: \cdots: 1]\} \subseteq \mathbb{P}^{N},
$$

and that for any $1 \leqslant i \leqslant N-2, A_{i}$ is the set of all $\left(\begin{array}{c}N+2 \\ i+1\end{array}\right)$ linear subspaces of dimension $i$ spanned by $i+1$ distinct points from $A_{0}$. The space $X$ was constructed as an iterated blow-up

$$
X:=X_{N-1} \stackrel{\alpha_{N-2}}{\longrightarrow} X_{N-2} \stackrel{\alpha_{N-3}}{\longrightarrow} \cdots \stackrel{\alpha_{2}}{\longrightarrow} X_{2} \stackrel{\alpha_{1}}{\longrightarrow} X_{1} \stackrel{\alpha_{0}}{\longrightarrow} X_{0},
$$

where $X_{0}=\mathbb{P}^{N}$, and for each $0 \leqslant i \leqslant N-2$ we have that $\alpha_{i}: X_{i+1} \rightarrow X_{i}$ is the blow-up of $X_{i}$ along the proper transform $\widetilde{A_{i}}$ of $A_{i}$ under $\alpha_{0} \circ \cdots \circ \alpha_{i-1}$.

The following lemma helps keep track of intersections between centers of the blow-ups. It is a restatement of [Ll, Lemma 3.2.3].

LeMmA 5.4. Let $L$ and $M$ be irreducible components of $A_{i}$ and $A_{j}$. Then either:

(1) $L \cap M=\emptyset$,

(2) $L \cap M$ is an irreducible component of $A_{k}$ for some $k \leqslant \min (i, j)$, or

(3) L intersects $M$ transversally.

In particular, if $L$ and $M$ are two distinct centers of the same dimension and (1) or (2) holds, then by the time we blow them up they will be disjoint. Otherwise, (3) holds and (since transversality is preserved under blowing up) the order of blow-ups will not matter, by Lemma 5.3.

Let $Y_{0}=X_{0}=\mathbb{P}^{N}$ and let $s_{0}: Y_{0} \rightarrow X_{0}$ be the squaring map

$$
s_{0}:\left[x_{1}: \cdots: x_{N+1}\right] \mapsto\left[x_{1}^{2}: \cdots: x_{N+1}^{2}\right] .
$$

For each $0 \leqslant i \leqslant N-2$, let $B_{i}:=s_{0}^{-1}\left(A_{i}\right)$ and $C_{i}:=B_{i}-A_{i}$. The space $Y$ is constructed by the following sequence of blow-ups:

$$
Y:=Y_{N-1} \stackrel{\beta_{N-2}}{\longrightarrow} Y_{N-2} \stackrel{\beta_{N-3}}{\longrightarrow} \cdots \stackrel{\beta_{2}}{\longrightarrow} Y_{2} \stackrel{\beta_{1}}{\longrightarrow} Y_{1} \stackrel{\beta_{0}}{\longrightarrow} Y_{0}
$$

where $\beta_{i}: Y_{i+1} \rightarrow Y_{i}$ is the blow-up of $Y_{i}$ along the proper transform $\widehat{B_{i}}$ of $B_{i}$ under $\beta_{0} \circ \cdots \circ \beta_{i-1}$.

Since properties (1)-(3) of Lemma 5.4 persist under taking inverse images by $s_{0}$, we also have the following lemma.

LEMMA 5.5. Let $L$ and $M$ be irreducible components of $B_{i}$ and $B_{j}$. Then either:

(1) $L \cap M=\emptyset$,

(2) $L \cap M$ is an irreducible component of $B_{k}$ for some $k \leqslant \min (i, j)$, or

(3) L intersects $M$ transversally.

Lemma 5.6. There exist maps $\mathrm{pr}: Y \rightarrow X$ and $\tilde{s}: Y \rightarrow X$ making diagram (6) commute (where $s \circ \mathrm{pr}$ is defined). 
Proof. Consider the diagram
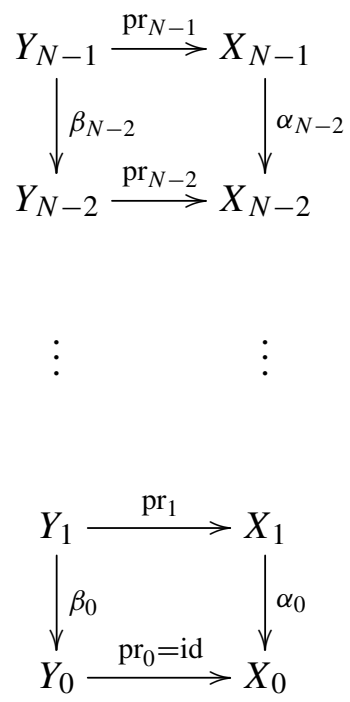

We will use induction to prove that for every $0 \leqslant i \leqslant N-1$ there are mappings $\mathrm{pr}_{i}: Y_{i} \rightarrow X_{i}$ making the diagram commute with the following two additional properties:

(1) for any $i \leqslant l \leqslant N-2$,

$$
D_{l}:=\overline{\operatorname{pr}_{i}^{-1}\left(\widetilde{A}_{l}\right) \backslash \widehat{A}_{l}}
$$

is a Cartier subscheme of $Y_{i}$, where tilde denotes proper transform under $\alpha_{i-1} \circ \cdots \circ \alpha_{0}$ and hat denotes proper transform under $\beta_{i-1} \circ \cdots \circ \beta_{0}$, and

(2) for every $i \leqslant l, m \leqslant N-2$, we have $\widehat{A}_{l} \nsubseteq D_{m}$.

Remark 5.7. When $N=2$ and therefore $i=0, D_{l}=\emptyset$. However, $D_{l}$ is typically nonempty, including the case of $N=3$ and $i=1$, where

$$
D_{1}=E_{[1:-1: 1: 1]} \cup E_{[1: 1:-1: 1]} \cup E_{[1: 1: 1:-1]} .
$$

These are the exceptional divisors obtained when blowing up the points of $B_{0} \cap A_{1}$. In general, $D_{l}$ can be thought of as the 'extra' exceptional divisors lying over $A_{l}$ produced in the construction of $Y_{i}$ that were not producted in the construction of $X_{i}$.

As the base case of the induction, notice that $\mathrm{pr}_{0}=\mathrm{id}: Y_{0} \rightarrow X_{0}$ trivially satisfies both (1) and (2).

We now suppose that there is a mapping $\mathrm{pr}_{i}: Y_{i} \rightarrow X_{i}$ for which properties (1) and (2) hold. We will use the universal property of blow-ups to construct $\mathrm{pr}_{i+1}: Y_{i+1} \rightarrow X_{i+1}$ for which properties (1) and (2) hold as well.

By Lemmas 5.5 and 5.3 we can perform the blow-ups of irreducible components of $\widehat{B}_{i}$ in any order we like; recall that $C_{i}:=B_{i} \backslash A_{i}$. Let us first blow up $\widehat{A}_{i}$ and then $\widehat{C}_{i}$, factoring $\beta_{i}$ as a composition $Y_{i+1} \stackrel{\mu_{i}}{\rightarrow} Z_{i+1} \stackrel{\lambda_{i}}{\rightarrow} Y_{i}$, where $\lambda_{i}$ is the blow-up along $\widehat{A}_{i}$ and $\mu_{i}$ is the 
further blow-up along $\widehat{C}_{i}$. Let $\eta_{i}:=\operatorname{pr}_{i} \circ \lambda_{i}$ and consider the diagram

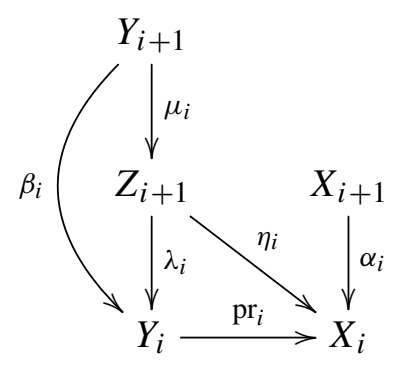

We will use the universal property to construct $q_{i+1}: Z_{i+1} \rightarrow X_{i+1}$ making the diagram commute. Then $\mathrm{pr}_{i+1}:=q_{i+1} \circ \mu_{i}$ will be the desired map.

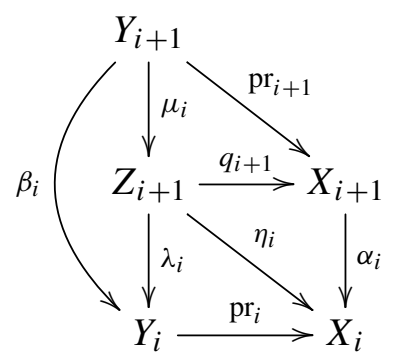

By the induction hypothesis, $\operatorname{pr}_{i}^{-1}\left(\widetilde{A}_{i}\right)=\widehat{A}_{i} \cup D_{i}$, where $D_{i}$ is an Cartier subscheme. By property (1) of the induction hypothesis,

$$
\eta_{i}^{-1}\left(\widetilde{A}_{i}\right)=\lambda_{i}^{-1}\left(\operatorname{pr}_{i}^{-1}\left(\widetilde{A}_{i}\right)\right)=\lambda_{i}^{-1}\left(\widehat{A}_{i} \cup D_{i}\right)=E_{\widehat{A}_{i}} \cup \lambda_{i}^{-1}\left(D_{i}\right)
$$

is a Cartier subscheme (where $E_{\widehat{A}_{i}}$ denotes the exceptional divisor). By the universal property of blow-ups, there exists a map $q_{i+1}: Z_{i+1} \rightarrow X_{i+1}$ making the diagram commute.

We must now check that $\operatorname{pr}_{i+1}:=q_{i+1} \circ \mu_{i}$ satisfies properties (1) and (2). We will first show that $q_{i+1}$ satisfies these properties. We will continue to use tildes to denote proper transforms living in $X_{i}$. When taking a further proper transform under $\alpha_{i}$, we will append '. Similarly, we will continue to use hats to denote proper transforms living in $Y_{i}$ and we will append ' to denote a further proper transform under $\lambda_{i}$ and " to denote a further proper transform under $\mu_{i}$.

Suppose that $i+1 \leqslant l \leqslant N-2$. Consider the proper transform of $\widetilde{A}_{l}$ under $\alpha_{i}$, which is given by $\widetilde{A}_{l}^{\prime}=\overline{\alpha_{i}^{-1}\left(\widetilde{A}_{l} \backslash \widetilde{A}_{i}\right)}$. Since $q_{i+1}: Z_{i+1} \rightarrow X_{i+1}$ is continuous and closed,

$$
\begin{aligned}
\left.\left(q_{i+1}\right)^{-1}\left(\widetilde{A}_{l}^{\prime}\right)=\left(q_{i+1}\right)^{-1} \overline{\left(\alpha_{i}^{-1}\left(\widetilde{A}_{l} \backslash \widetilde{A}_{i}\right)\right.}\right) & =\overline{\left(\alpha_{i} \circ q_{i+1}\right)^{-1}\left(\widetilde{A}_{l} \backslash \widetilde{A}_{i}\right)} \\
& =\overline{\lambda_{i}^{-1} \circ \operatorname{pr}_{i}^{-1}\left(\widetilde{A}_{l} \backslash \widetilde{A}_{i}\right)},
\end{aligned}
$$

using commutativity of (9). By the induction hypothesis, $\operatorname{pr}_{i}^{-1}\left(\widetilde{A}_{l}\right)=\widehat{A}_{l} \cup D_{l}$ and $\operatorname{pr}_{i}^{-1}\left(\widetilde{A}_{i}\right)=\widehat{A}_{i} \cup D_{i}$ with $D_{i}$ and $D_{l}$ both Cartier subschemes and $A_{l} \nsubseteq D_{i}$. We have

$$
\operatorname{pr}_{i}^{-1}\left(\widetilde{A}_{l} \backslash \widetilde{A}_{i}\right)=\left(\widehat{A}_{l} \cup D_{l}\right) \backslash\left(\widehat{A}_{i} \cup D_{i}\right)=\left(\widehat{A}_{l} \backslash\left(\widehat{A}_{i} \cup D_{i}\right)\right) \cup\left(\left(D_{l} \backslash D_{i}\right) \backslash \widehat{A}_{i}\right) .
$$

Since $\widehat{A}_{l} \nsubseteq D_{i}$, we have that

$$
\overline{\lambda_{i}^{-1}\left(\widehat{A}_{l} \backslash\left(\widehat{A}_{i} \cup D_{i}\right)\right)}=\widehat{A}_{l}^{\prime}
$$


Meanwhile, since $D_{l}$ and $D_{i}$ are Cartier subschemes of $Y_{i}$,

$$
H_{l}:=\overline{\lambda_{i}^{-1}\left(\left(D_{l} \backslash D_{i}\right) \backslash \widehat{A}_{i}\right)} \subseteq Z_{i+1}
$$

is a (potentially empty) Cartier subscheme. Thus,

$$
\left(q_{i+1}\right)^{-1}\left(\widetilde{A}_{l}^{\prime}\right)=\overline{\lambda_{i+1}^{-1} \circ \operatorname{pr}_{i}^{-1}\left(\widetilde{A}_{l} \backslash \widetilde{A}_{i+1}\right)}=\widehat{A}_{l}^{\prime} \cup H_{l}
$$

By the induction hypothesis, we have that for all $i+1 \leqslant l, m \leqslant N-2, \widehat{A_{l}} \not \nsubseteq D_{m}$ so that $\widehat{A}_{l} \cap D_{m}$ is a proper subvariety of $\widehat{A}_{l}$. Since $\widehat{A}_{i}$ is of lower dimension than $\widehat{A}_{l}$, there is a point $y \in \widehat{A_{l}} \backslash\left(\widehat{A}_{i} \cup D_{m}\right)$. Since $\lambda_{i}$ is surjective, any element of $\lambda_{i}^{-1}(y)$ gives a point of $\widehat{A}_{l}^{\prime} \backslash H_{m}$. Thus, $\widehat{A}_{l}^{\prime} \nsubseteq H_{m}$.

We will now pull everything back via the total transform under $\mu_{i}$ and check that properties (1) and (2) hold for $\operatorname{pr}_{i+1}:=q_{i+1} \circ \mu_{i}$. Consider any $i+1 \leqslant l \leqslant N-2$. It follows from Lemma 5.4 that for any irreducible components $L$ of $\widehat{C}_{i}^{\prime}$ and $M$ of $\widehat{A}_{l}^{\prime}$ we have that either $L \cap M=\emptyset, L$ and $M$ are transverse, or $L \subseteq M$. In the first case, the total transform of $M$ under the blow-up of $L$ coincides with the proper transform $M^{\prime \prime}$. This also holds in the second case, by Lemma 5.3. In the last case, the total transform of $M$ is $M^{\prime} \cup E_{L}$, where $E_{L}$ is the exceptional divisor over $L$. Therefore,

$$
\mu_{i+1}^{-1}\left(\widehat{A}_{l}^{\prime}\right)=\widehat{A}_{l}^{\prime \prime} \cup E_{l},
$$

where $E_{l}$ is the union of exceptional divisors over the components of $\widehat{C}_{i}^{\prime}$ lying entirely within $\widehat{A}_{l}^{\prime}$. Meanwhile,

$$
K_{l}:=\mu_{i}^{-1}\left(H_{l}\right)
$$

is a Cartier subscheme. Thus,

$$
\operatorname{pr}_{i+1}^{-1}\left(\widetilde{A}_{l}^{\prime}\right)=\mu_{i}^{-1}\left(\widehat{A}_{l}^{\prime} \cup H_{l}\right)=\widehat{A}_{l}^{\prime \prime} \cup E_{l} \cup K_{l}
$$

where $E_{l} \cup K_{l}$ is a Cartier subscheme. In particular, property (1) holds.

To see that property (2) holds, notice that for any $i+1 \leqslant l, m \leqslant N-2$ we have $\widehat{A}_{l}^{\prime \prime} \nsubseteq E_{\widehat{C}_{i}}$ since $\widehat{A}_{l}$ is of greater dimension than $\widehat{C}_{i}^{\prime}$. Taking a point $y \in \widehat{A}_{l} \backslash\left(\widehat{C}_{i}^{\prime} \cup H_{m}\right)$, we see that $\mu_{i}^{-1}(y)$ is a non-empty subset of $\widehat{A}_{l}^{\prime \prime} \backslash\left(E_{m} \cup K_{m}\right)$. Thus, $\widehat{A}_{l}^{\prime \prime} \nsubseteq\left(E_{m} \cup K_{m}\right)$, establishing that property (2) holds.

By induction, we conclude that for each $0 \leqslant i \leqslant N-1$ there exist mappings $\mathrm{pr}_{i}: Y_{i} \rightarrow X_{i}$ making diagram (7) commute.

We will now construct the map $\widetilde{s}: Y \rightarrow X$. Let $s_{0}: \mathbb{P}^{N} \rightarrow \mathbb{P}^{N}$ be the squaring map. Since $B_{0}=s_{0}^{-1}\left(A_{0}\right)$, Corollary 5.2 gives that $s_{0} \equiv \widetilde{s}_{0}: Y_{0} \rightarrow X_{0}$ lifts to a holomorphic map $\tilde{s}_{1}: Y_{1} \rightarrow X_{1}$ :

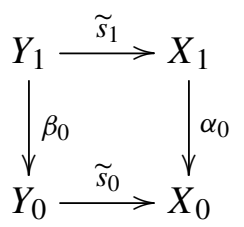


Notice that $\widehat{B}_{1}=\left(\widetilde{s}_{1}\right)^{-1}\left(\widetilde{A}_{1}\right)$, so that we can again apply Corollary 5.2 to lift $\widetilde{s}_{1}$ to a holomorphic map $\widetilde{s}_{2}: Y_{2} \rightarrow X_{2}$ making the following diagram commute:

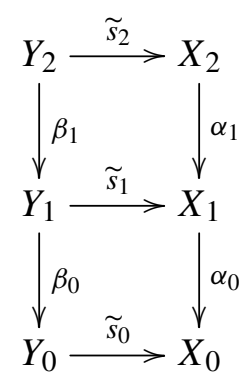

Continuing in this way, we obtain holomorphic maps $\widetilde{s}_{i}: Y_{i} \rightarrow X_{i}$ for $1 \leqslant i \leqslant N-1$ making the following diagram commute:
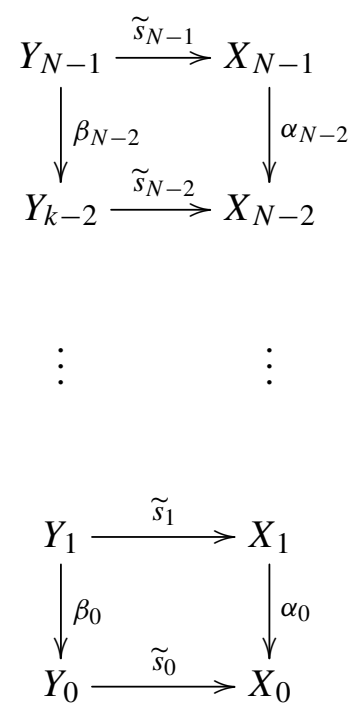

The desired map is $\widetilde{s} \equiv \widetilde{s}_{N-1}: Y_{N-1} \rightarrow X_{N-1}$.

We must now check that diagram (6) commutes wherever $s \circ$ pr is defined, that is, on $Y \backslash \mathrm{pr}^{-1}\left(I_{S}\right)$. Since $Y$ is connected, it suffices to prove commutativity on any open subset of $Y \backslash \mathrm{pr}^{-1}\left(I_{S}\right)$. Let

$$
\mathcal{A}:=\alpha_{0} \circ \cdots \circ \alpha_{N-2}: X \rightarrow \mathbb{P}^{N} \text { and } \mathcal{B}=\beta_{0} \circ \cdots \circ \beta_{N-2}: Y \rightarrow \mathbb{P}^{N}
$$

be the compositions of the blow-ups used to construct $X$ and $Y$. Consider an open subset $U \subseteq \mathbb{P}^{N}$ with $U$ disjoint from $\bigcup_{i=0}^{N-2} B_{i}$. Then, $\left.\mathcal{B}\right|_{\mathcal{B}^{-1}(U)}: \mathcal{B}^{-1}(U) \rightarrow U$ and $\left.\mathcal{A}\right|_{\mathcal{A}^{-1}(U)}: \mathcal{A}^{-1}(U) \rightarrow U$ serve as local coordinate charts on $Y$ and $X$. Commutativity of (7) gives that when pr is expressed in these coordinates it becomes the identity.

Since $V:=\widetilde{s}_{0}(U)$ is disjoint from $\bigcup_{i=0}^{N-2} A_{i}$, we have that $\left.\mathcal{A}\right|_{\mathcal{A}^{-1}(V)}: \mathcal{A}^{-1}(V) \rightarrow V$ serves as a local coordinate chart on $X$. Commutativity of (11) implies that when expressed in the $\left.\mathcal{B}\right|_{\mathcal{B}^{-1}(U)}$ and $\left.\mathcal{A}\right|_{\mathcal{A}^{-1}(V)}$ coordinates, $\widetilde{s}$ is given by $\widetilde{s}_{0}: U \rightarrow V$.

By definition, when $s: X \rightarrow X$ is expressed in the $\left.\mathcal{B}\right|_{\mathcal{B}^{-1}(U)}$ and $\left.\mathcal{A}\right|_{\mathcal{A}^{-1}(V)}$ coordinates, it becomes $\widetilde{s}_{0}: U \rightarrow V$. Therefore, when expressed in the $\left.\mathcal{B}\right|_{\mathcal{B}^{-1}(U)}$ and $\left.\mathcal{A}\right|_{\mathcal{A}^{-1}(V)}$ coordinates $s \circ \mathrm{pr}$ is also given by $\widetilde{s}_{0}: U \rightarrow V$. We conclude that (6) commutes wherever $s \circ \mathrm{pr}$ is defined. 
LemMA 5.8. Let $\widetilde{s}: Y \rightarrow X$ be the map constructed above. For every $x \in X$ the $\operatorname{set}^{-1}(x)$ is finite.

The proof of this lemma was inspired by techniques of Lloyd-Philipps [LI].

Proof. The proof will proceed by induction on the dimension $N$. In addition to using superscripts to index the dimension of the spaces $X^{N}$ and $Y^{N}$, we will also occasionally append them to our maps in order to specify the dimension of the spaces in the domain and codomain of the maps. For example, the superscript on $\widetilde{s}^{N}$ indicates that it is a mapping $\widetilde{s}^{N}: Y^{N} \rightarrow X^{N}$ and the superscript on $A_{l}^{N}$ indicates that it is a subset of $\mathbb{P}^{N}$.

For the inductive proof, it will be helpful to consider the one-point spaces $\mathbb{P}^{0}, X^{0}$, and $Y^{0}$ for which it is trivial that $\widetilde{s}^{0}: Y^{0} \rightarrow X^{0}$ has finite fibers. When $N=1$ we have $Y^{1} \equiv$ $Y_{0}^{1} \equiv \mathbb{P}^{1}$ and $X^{1} \equiv X_{0}^{1} \equiv \mathbb{P}^{1}$ and $\widetilde{s}^{1} \equiv \widetilde{s}_{0}^{1}: \mathbb{P}^{1} \rightarrow \mathbb{P}^{1}$ is the squaring map, which clearly has finite fibers.

Now suppose that for each $1 \leqslant i<N$, the mappings $\widetilde{s}^{i}: Y^{i} \rightarrow X^{i}$ have finite fibers in order to prove that $\widetilde{s}^{N}: Y^{N} \rightarrow X^{N}$ has finite fibers.

Recall the commutative diagram

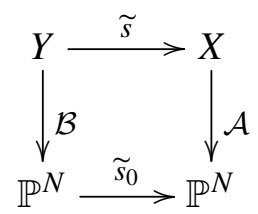

where $\mathcal{A}$ and $\mathcal{B}$ are the compositions of blow-ups defined in (12). Let $z=\mathcal{A}(x)$ and notice that since the squaring map $\widetilde{s}_{0}$ has finite fibers, there are finitely many points $w \in \widetilde{s}_{0}^{-1}(z) \subset \mathbb{P}^{N}$ over which the preimages $\widetilde{s}^{-1}(x)$ lie. Thus, for any such $z$ and $w$ it suffices show that

$$
\left.\widetilde{s}\right|_{\mathcal{B}^{-1}(w)}: \mathcal{B}^{-1}(w) \rightarrow \mathcal{A}^{-1}(z)
$$

has finite fibers.

If $\mathcal{A}(x)$ is not a critical value of $\widetilde{s_{0}}$, then for any $y \in \widetilde{s}^{-1}(x)$ there is a neighborhood $U$ of $\mathcal{B}(y)$ so that $\widetilde{s}_{0}: U \rightarrow \widetilde{s}_{0}(U)=: V$ is a biholomorphism. Iteratively applying Corollary 5.2 to $\widetilde{s}_{0}$ and its inverse gives that $\widetilde{s}: \mathcal{B}^{-1}(U) \rightarrow \mathcal{A}^{-1}(V)$ is a biholomorphism.

If $\mathcal{A}(x)$ is a critical value, the proof is more subtle. We will use the recursive structure of $X^{N}$ and $Y^{N}$ in order to express these fibers as products of lower-dimensional $X^{i}$ and $Y^{i}$, which will allow us to express (14) in terms of $\widetilde{s}^{i}: Y^{i} \rightarrow X^{i}$ and $\operatorname{id}^{i}: X^{i} \rightarrow X^{i}$, for $0 \leqslant i<N$, where $\mathrm{id}^{i}$ is the identity mapping.

Notice that the construction of $\widetilde{s}: Y \rightarrow X$ commutes with permutations

$$
\sigma:\left[z_{1}: z_{2}: \cdots: z_{N+1}\right] \mapsto\left[z_{\sigma(1)}: z_{\sigma(2)}: \cdots: z_{\sigma(N+1)}\right]
$$

of the coordinates on $\mathbb{P}^{N}$. In particular, we can suppose without loss of generality that $z=\mathcal{A}(x)=\left[0: \cdots: 0: z_{l+1}: \cdots: z_{N+1}\right]$ with $z_{i} \neq 0$ for $l+1 \leqslant i \leqslant N+1$ and that the remaining $z_{i}$ are grouped so that repeated values come in blocks. (Note that one can have $l=0$.) Commutative diagram (13) implies that $w=\left[0: \cdots: 0: w_{l+1}: \cdots: w_{N+1}\right]$, with $w_{i} \neq 0$ for $l+1 \leqslant i \leqslant N+1$. 
We need a more precise description of centers of the blow-ups $A_{l}^{N}$. Let

$$
q_{1}=[1: 0: \cdots: 0], \ldots, q_{N+1}=[0: \cdots: 0: 1], q_{N+2}=[1: 1: \cdots: 1] \in \mathbb{P}^{N},
$$

and for any $\left\{i_{1}, \ldots, i_{m+1}\right\} \subseteq\{1, \ldots, N+2\}$, let

$$
\Pi_{i_{1}, \ldots, i_{m+1}}:=\operatorname{span}\left(q_{i_{1}}, \ldots, q_{i_{m+1}}\right) \subseteq \mathbb{P}^{N} .
$$

Note that

$$
A_{m}=\bigcup_{\left\{i_{1}, \ldots, i_{m+1}\right\}} \Pi_{i_{1}, \ldots, i_{m+1}}
$$

where the union is taken over all subsets $\left\{i_{1}, \ldots, i_{m+1}\right\} \subseteq\{1, \ldots, N+2\}$.

We will need a more precise description of which components of $z$ are equal. Let us suppose that

$$
\begin{aligned}
z_{l+1} & =z_{l+2}=\cdots=z_{l+j_{1}}, \\
z_{l+j_{1}+1} & =z_{l+j_{1}+2}=\cdots=z_{l+j_{1}+j_{2}}, \\
& \vdots \\
z_{l+j_{1}+\cdots+j_{a-1}+1} & =z_{l+j_{1}+\cdots+j_{a-1}+2}=\cdots=z_{l+j_{1}+\cdots+j_{a}},
\end{aligned}
$$

with no equality between any pair of lines. In other words, the first $j_{1}$ non-zero entries are equal, the next $j_{2}$ non-zero entries are equal and distinct from the first $j_{1}$ non-zero entries, etc. We assume that each $j_{1}, \ldots, j_{a} \geqslant 2$ and that all values appearing in the remaining components of $z$ occur only once.

We will show that

$$
\begin{aligned}
& \mathcal{A}^{-1}(z) \cong X^{l-1} \times X^{j_{1}-2} \times \cdots \times X^{j_{a}-2} \\
& \mathcal{B}^{-1}(w) \cong Y^{l-1} \times X^{j_{1}-2} \times \cdots \times X^{j_{a}-2}
\end{aligned}
$$

and that (in the coordinates given by these isomorphisms)

$$
\left.\widetilde{s}\right|_{\mathcal{B}^{-1}(w)}=\widetilde{s}^{l-1} \times \mathrm{id}^{j_{1}-2} \times \cdots \times \mathrm{id}^{j_{a}-2},
$$

where $\mathrm{id}^{i}: X^{i} \rightarrow X^{i}$ denotes the identity mapping. By the induction hypothesis, (17) will imply that $\left.\widetilde{s}\right|_{\mathcal{B}^{-1}(w)}: \mathcal{B}^{-1}(w) \rightarrow \mathcal{A}^{-1}(z)$ has finite fibers and thus complete the proof.

We will first check that (15) holds. Let $V \subseteq \mathbb{P}^{N}$ be a neighborhood of $z$ chosen small enough so that it intersects $\Pi_{i_{1}, \ldots, i_{m+1}}$ if and only if $z \in \Pi_{i_{1}, \ldots, i_{m+1}}$. In order to study $\mathcal{A}^{-1}(z)$ we will work with $\mathcal{A}^{-1}(V)$.

Associated to the particular points $z \in \mathbb{P}^{N}$ above, we have the following sets. Let $S=$ $\{1, \ldots, N+2\}$, and let

$$
\begin{aligned}
S^{0} & :=S \backslash\{1, \ldots, l, N+2\}, \\
S^{1} & :=S \backslash\left\{l+1, \ldots, l+j_{1}\right\}, \\
S^{2} & :=S \backslash\left\{l+j_{1}+1, \ldots, l+j_{1}+j_{2}\right\}, \\
& \vdots \\
S^{a} & :=S \backslash\left\{l+j_{1}+\cdots+j_{a-1}, \ldots, l+j_{1}+\cdots+j_{a}\right\} .
\end{aligned}
$$


Note that $z \in \Pi_{i_{1}, \ldots, i_{m+1}}$ if and only if $S^{b} \subseteq\left\{i_{1}, \ldots, i_{m+1}\right\}$ for some $0 \leqslant b \leqslant a$. We will call each of the centers $\Pi_{S^{b}}$ for $0 \leqslant b \leqslant a$ primitive center since any center of blow-up through $z$ will contain at least one of them. Since $S_{b} \cup S_{c}=S$ for any $b \neq c$, any center through $z$ that is blown up contains a unique primitive center. Thus, any further center that is blown up is of the form

$$
\Pi_{T}, \quad \text { where } T=S^{b} \cup\left\{i_{0}, \ldots, i_{m}\right\} .
$$

We will call $\Pi_{T}$ subordinate to $\Pi_{S^{b}}$.

Since $S_{b} \cup S_{c}=S$ for $b \neq c$, it also follows that any center subordinate to $\Pi_{S^{b}}$ is transverse to any center subordinate to $\Pi_{S^{c}}$. Since blow-ups preserve transversality, this will also hold for the proper transforms. Therefore, by Lemma 5.3, we can exchange the order of blow-up between two centers subordinate to distinct primitive centers and still get the same result for $\mathcal{A}^{-1}(V)$. In particular, we can first blow up each of the primitive centers. After doing so, we can blow up all of the (proper transforms of) centers subordinate to $\Pi_{S^{0}}$, by order of increasing dimension. We can then blow up all (proper transforms of) centers subordinate to $\Pi_{S^{1}}$ by order of increasing dimension, etc.

Let $\left[v_{1}: v_{2}: \cdots: v_{N+1}\right]$ be homogeneous coordinates on $\mathbb{P}^{N}$. Blowing up $\Pi_{S^{0}}$ produces

$$
\left\{\left[v_{1}: v_{2}: \cdots: v_{N+1}\right] \times\left[m_{1}^{0}: \cdots: m_{l}^{0}\right] \in V \times \mathbb{P}^{l}:\left(v_{1}, \ldots, v_{l}\right) \sim\left(m_{1}^{0}, \ldots, m_{l}^{0}\right)\right\},
$$

where $\sim$ indicates that one vector is a multiple of another.

Blowing up each of the remaining primitive centers $\Pi_{S^{1}}, \ldots, \Pi_{S^{a}}$ produces the subset of $V \times \mathbb{P}^{l-1} \times \mathbb{P}^{j_{1}-2} \times \cdots \times \mathbb{P}^{j_{a}-2}$ given in the coordinates

$$
\left\{\left[v_{1}: \cdots: v_{N+1}\right] \times\left[m_{1}^{0}: \cdots: m_{l}^{0}\right] \times\left[m_{1}^{1}: \cdots: m_{j_{1}-1}^{1}\right] \times \cdots \times\left[m_{1}^{a}: \cdots: m_{j_{a}-1}^{a}\right]\right.
$$

by the conditions

$$
\begin{aligned}
\left(m_{1}^{0}, \ldots, m_{l}^{0}\right) & \sim\left(v_{1}, \ldots, v_{l}\right) \\
\left(m_{1}^{1}, \ldots, m_{j_{1}-1}^{1}\right) \sim & \left(v_{l+2}-v_{l+1}, v_{l+3}-v_{l+1}, \ldots, v_{l+j_{1}}-v_{l+1}\right) \\
& \vdots \\
\left(m_{1}^{a}, \ldots, m_{j_{a}-1}^{a}\right) \sim & \left(v_{l+j_{1}+\cdots+j_{a-1}+2}-v_{l+j_{1}+\cdots+j_{a-1}+1}, \ldots, v_{l+j_{1}+\cdots+j_{a}}\right. \\
& \left.\quad-v_{l+j_{1}+\cdots+j_{a-1}+1}\right) .
\end{aligned}
$$

Let us denote this blow-up at all of the primitive centers by $v: V^{\#} \rightarrow V$. The fiber over $z$ is $v^{-1}(z) \cong \mathbb{P}^{l-1} \times \mathbb{P}^{j_{1}-2} \times \cdots \times \mathbb{P}^{j_{a}-2}$. We will now check that blow-ups along the proper transforms of the centers subordinate to $\Pi_{S^{0}}, \ldots, \Pi_{S^{a}}$ result in suitable blow-ups of $v^{-1}(z)$ in order to transform it into $X^{l-1} \times X^{j_{1}-2} \times \cdots \times X^{j_{a}-2}$.

Each of the centers subordinate to $\Pi_{S^{0}}$ will be of the form $\Pi_{T}$ where $T=S^{0} \cup$ $\left\{i_{1}, \ldots, i_{m}\right\}$, for $\left\{i_{1}, \ldots, i_{m}\right\} \subseteq\{1, \ldots, l, N+2\}$. There are precisely $l+1$ centers of dimension one greater than the dimension of $\Pi_{S^{0}}$; they are

$$
\Pi_{S^{0} \cup\{1\}}, \ldots, \Pi_{S^{0} \cup\{l\}}, \Pi_{S^{0} \cup\{N+2\}} \cdot
$$


One can check that the proper transforms of these intersect $\mathbb{P}^{l-1} \times \mathbb{P}^{j_{1}-2} \times \cdots \times \mathbb{P}^{j_{a}-2}$ at

$$
\begin{gathered}
\{[1: 0: \cdots: 0]\} \times \mathbb{P}^{j_{1}-2} \times \cdots \times \mathbb{P}^{j_{a}-2}, \\
\vdots \\
\{[0: 0: \cdots: 1]\} \times \mathbb{P}^{j_{1}-2} \times \cdots \times \mathbb{P}^{j_{a}-2}, \quad \text { and } \\
\{[1: 1: \cdots: 1]\} \times \mathbb{P}^{j_{1}-2} \times \cdots \times \mathbb{P}^{j_{a}-2},
\end{gathered}
$$

respectively. In other words, the centers of dimension one greater than $\Pi_{S^{0}}$ that are subordinate to $\Pi_{S^{0}}$ intersect $v^{-1}(z)$ in $A_{0}^{l-1} \times \mathbb{P}^{j_{1}-2} \times \cdots \times \mathbb{P}^{j_{a}-2}$.

If we let

$$
\hat{q}_{1}=[1: 0: \cdots: 0], \ldots, \hat{q}_{l}=[0: 0: \cdots: 1], \hat{q}_{l+1}=[1: 1: \cdots: 1] \in \mathbb{P}^{l-1},
$$

then for any $\left\{i_{1}, \ldots, i_{m}\right\} \subseteq\{1, \ldots, l, N+2\}$, one can check that the proper transform of

$$
\Pi_{S^{0} \cup\left\{i_{1}, \ldots, i_{m}\right\}}
$$

intersects $v^{-1}(z)$ in $\hat{\Pi}_{i_{0}, \ldots, i_{m}} \times \mathbb{P}^{j_{1}-2} \times \cdots \times \mathbb{P}^{j_{a}-2}$, where

$$
\hat{\Pi}_{i_{1}, \ldots, i_{m}}=\operatorname{span}\left\{\hat{q}_{i_{1}}, \ldots, \hat{q}_{i_{m}}\right\} \subseteq \mathbb{P}^{l-1} .
$$

In particular, for any $1 \leqslant b \leqslant l-1$, the centers of dimension $b$ greater than the dimension of $\Pi_{S^{0}}$ that are subordinate to $\Pi_{S^{0}}$ intersect $v^{-1}(z)$ in $A_{b-1}^{l-1} \times \mathbb{P}^{j_{1}-2} \times \cdots \times \mathbb{P}^{j_{a}-2}$.

Therefore, blowing up all of the centers subordinate to $\Pi_{S^{0}}$ in order of increasing dimension results in a sequential blow-up of the first factor $\mathbb{P}^{l-1}$ transforming it into $X^{l}$. It leaves each of the remaining factors unchanged.

Matters are almost the same for the remaining factors. Let us illustrate the only difference by discussing the second factor $\mathbb{P}^{j_{1}-2}$. Each of the centers subordinate to $\Pi_{S^{1}}$ will be of the form

$$
\Pi_{S^{1} \cup\left\{i_{1}, \ldots, i_{m}\right\}}, \quad \text { where }\left\{i_{1}, \ldots, i_{m}\right\} \subseteq\left\{l+1, \ldots, l+j_{1}\right\} .
$$

Thus there are $j_{1}$ centers of dimension one greater:

$$
\Pi_{S^{1} \cup\{l+1\}}, \ldots, \Pi_{S^{1} \cup\left\{l+j_{1}\right\}} .
$$

One can check that their proper transforms intersect $v^{-1}(z)$ in

$$
\begin{gathered}
\mathbb{P}^{l-1} \times\{[1: 1: \cdots: 1]\} \times \mathbb{P}^{j_{2}-2} \times \cdots \times \mathbb{P}^{j_{a}-2}, \\
\mathbb{P}^{l-1} \times\{[1: 0: \cdots: 0]\} \times \mathbb{P}^{j_{2}-2} \times \cdots \times \mathbb{P}^{j_{a}-2}, \quad \text { and } \\
\vdots \\
\mathbb{P}^{l-1} \times\{[0: 0: \cdots: 1]\} \times \mathbb{P}^{j_{2}-2} \times \cdots \times \mathbb{P}^{j_{a}-2},
\end{gathered}
$$

respectively. Using similar reasoning to that from the analysis of the first factor, we can see that the centers of dimension $b$ greater than $\Pi_{S^{1}}$ that are subordinate to $\Pi_{S^{1}}$ will intersect $v^{-1}(z)$ in $\mathbb{P}^{l-1} \times A_{b-1}^{j_{1}-2} \times \mathbb{P}^{j_{1}-2} \times \cdots \times \mathbb{P}^{j_{a}-2}$. In particular, blowing up all centers subordinate to $\Pi_{S^{1}}$ in order of dimension will result in blowing up the second factor from $\mathbb{P}^{j_{1}-2}$ to $X^{j_{1}-2}$. 
We conclude that (15) holds.

We will now prove (16) and (17). Let $U$ be the component of $\widetilde{s}_{0}^{-1}(V)$ containing $w$. We will study $\mathcal{B}^{-1}(U)$ in order to understand $\mathcal{B}^{-1}(w)$ and $\left.\widetilde{s}\right|_{\mathcal{B}^{-1}(w)}$.

Each of the centers $B_{i}$ that are blown up in the construction of $Y$ are obtained as preimages of the centers $A_{i}$ under $\widetilde{s}_{0}$. In particular, the only centers that will be blown up to construct $\mathcal{B}^{-1}(U)$ are the preimages of the centers subordinate to the primitive centers $\Pi_{S^{0}}, \ldots, \Pi_{S^{a}}$.

Each point $q_{1}, \ldots, q_{N+1}$ is totally invariant under $\widetilde{s}_{0}$ so that there are no additional preimages of them. Meanwhile, $q_{N+2}$ has $2^{N}$ preimages, consisting of all points of the form $[1: \pm 1: \pm 1: \cdots: \pm 1]$. Each of the centers from $B_{i}$ is the span of $i+1$ of these $N+1+2^{N}$ points.

Each primitive center $\Pi_{S^{0}}, \ldots, \Pi_{S^{a}}$ has a unique preimage under $\widetilde{s}_{0}$ that contains the point $w$ (as can be explicitly verified). Let $\Lambda^{0}, \ldots, \Lambda^{a}$ be the unique preimages of the primitive centers that contain $w$. Each of the further centers that is blown up will be subordinate to one of these primitive centers and those subordinate to distinct primitive centers intersect transversally. In particular, we can blow up to form $\mathcal{B}^{-1}(U)$ in precisely the same order as we did to form $\mathcal{A}^{-1}(V)$.

Let us first blow up the primitive centers, replacing $U$ by the subset of $U \times \mathbb{P}^{l-1} \times$ $\mathbb{P}^{j_{1}-2} \times \cdots \times \mathbb{P}^{j_{a}-2}$ given in the coordinates

$$
\left\{\left[u_{1}: u_{2}: \cdots: u_{N+1}\right] \times\left[n_{1}^{0}: \cdots: n_{l}^{0}\right] \times\left[n_{1}^{1}: \cdots: n_{j_{1}-1}^{1}\right] \times \cdots \times\left[n_{1}^{a}: \cdots: n_{j_{a}-1}^{a}\right]\right.
$$

by

$$
\begin{aligned}
\left(n_{1}^{0}, \ldots, n_{l}^{0}\right) & \sim\left(u_{1}, \ldots, u_{l}\right), \\
\left(n_{1}^{1}, \ldots, n_{j_{1}-1}^{1}\right) & \sim\left(u_{l+2} \pm u_{l+1}, u_{l+3} \mp u_{l+1}, \ldots, u_{l+j_{1}} \mp u_{l+1}\right) \\
& \vdots \\
\left(n_{1}^{j}, \ldots, n_{j_{a}-1}^{j}\right) & \sim\left(u_{l+j_{1}+\cdots+j_{a-1}+2} \mp u_{l+j_{1}+\cdots+j_{a-1}+1}, \ldots, u_{l+j_{1}+\cdots+j_{a}}\right. \\
& \left.\left.\mp u_{l+j_{1}+\cdots+j_{a-1}+1}\right)\right\} .
\end{aligned}
$$

Let us denote the blow-up of $U$ along all of the primitive centers $\Lambda^{0}, \ldots, \Lambda^{a}$ by $\mu: U^{\#} \rightarrow U$. In particular, the fiber over $w$ is $\mu^{-1}(w) \cong \mathbb{P}^{l-1} \times \mathbb{P}^{j_{1}-2} \times \cdots \times \mathbb{P}^{j_{a}-2}$.

Notice that $\tilde{s}_{0}: U \rightarrow V$ is given by

$$
\left[v_{1}: \cdots: v_{N+1}\right]=\widetilde{s}_{0}\left(\left[u_{1}: \cdots: u_{N+1}\right]\right)=\left[u_{1}^{2}: \cdots: u_{N+1}^{2}\right] .
$$

By Corollary 5.2, this lifts to a holomorphic mapping $s^{\#}: U^{\#} \rightarrow V^{\#}$ whose restriction $\left.s^{\#}\right|_{\mu^{-1}(w)}: \mu^{-1}(w) \rightarrow v^{-1}(z)$ is given by

$$
\begin{aligned}
& \left.s^{\#}\right|_{\mu^{-1}(w)}\left(\left[n_{1}^{0}: \cdots: n_{l}^{0}\right],\left[n_{1}^{1}: \cdots: n_{j_{1}-1}^{1}\right], \ldots,\left[n_{1}^{k}: \cdots: n_{j_{a}-1}^{j}\right]\right) \\
& \quad=\left(\left[\left(n_{1}^{0}\right)^{2}: \cdots:\left(n_{l}^{0}\right)^{2}\right],\left[n_{1}^{1}: \cdots: n_{j_{1}-1}^{1}\right], \ldots,\left[n_{1}^{k}: \cdots: n_{j_{a}-1}^{j}\right]\right) .
\end{aligned}
$$

In other words, the restriction $\left.s^{\#}\right|_{\mu^{-1}(w)}: \mu^{-1}(w) \rightarrow v^{-1}(z)$ is the squaring map on the first factor and the identity on each of the remaining factors.

We now blow up all of the centers that are subordinate to $\Lambda^{0}$. They are preimages under $\widetilde{s}_{0}$ of the centers subordinate to $\Pi_{S^{0}}$. In particular, the places where their proper transforms 
intersect $\mu^{-1}(w)$ are obtained as the preimages under $s^{\#}$ of the places where the centers subordinate to $\Pi_{S^{0}}$ intersect $v^{-1}(z)$. Thus, for all $0 \leqslant i \leqslant l-3$,

$$
B_{i}^{l-1} \times \mathbb{P}^{j_{1}-2} \times \cdots \times \mathbb{P}^{j_{a}-2}=\left(s^{\#}\right)^{-1}\left(A_{i}^{l-1} \times \mathbb{P}^{j_{1}-2} \times \cdots \times \mathbb{P}^{j_{a}-2}\right) .
$$

Blowing these centers up in order of dimension modifies $\mu^{-1}(w)$ to become

$$
Y^{l-1} \times \mathbb{P}^{j_{1}-2} \times \cdots \times \mathbb{P}^{j_{a}-2},
$$

and the map $s^{\#}$ lifts to a holomorphic map

$$
\widetilde{s}^{\#}: Y^{l-1} \times \mathbb{P}^{j_{1}-2} \times \cdots \times \mathbb{P}^{j_{a}-2} \rightarrow X^{l-1} \times \mathbb{P}^{j_{1}-2} \times \cdots \times \mathbb{P}^{j_{a}-2}
$$

whose action on the first term in the Cartesian product is $\widetilde{s}^{l-1}: Y^{l-1} \rightarrow X^{l-1}$ (by the uniqueness in Corollary 5.2). The action on each of the remaining terms of the product is the identity.

We now blow up the centers that are subordinate to $\Lambda^{1}$. They are preimages under $\widetilde{s}_{0}$ of the centers subordinate to $\Pi_{S^{1}}$. In particular, the places where their proper transforms intersect the fiber over $w$ are obtained as the preimages under $\widetilde{s}^{\#}$ of the places where the centers subordinate to $\Pi_{S^{1}}$ intersect the fiber over $z$. Thus, for all $0 \leqslant i \leqslant j_{1}-3$,

$$
Y^{l-1} \times A_{i}^{j_{1}-2} \times \cdots \times \mathbb{P}^{j_{a}-2}=\left(\widetilde{s}^{\#}\right)^{-1}\left(X^{l-1} \times A_{i}^{j_{1}-2} \times \cdots \times \mathbb{P}^{j_{a}-2}\right) .
$$

Blowing these centers up in order of dimension modifies the fiber over $w$ to become

$$
Y^{l-1} \times X^{j_{1}-2} \times \mathbb{P}^{j_{2}-2} \times \cdots \times \mathbb{P}^{j_{a}-2},
$$

and the map $\widetilde{s}^{\#}$ lifts to a holomorphic map

$$
\widehat{s}^{\#}: Y^{l-1} \times X^{j_{1}-2} \times \mathbb{P}^{j_{2}-2} \times \cdots \times \mathbb{P}^{j_{a}-2} \rightarrow X^{l-1} \times X^{j_{1}-2} \times \mathbb{P}^{j_{2}-2} \times \cdots \times \mathbb{P}^{j_{a}-2}
$$

whose action on first term in the Cartesian product remains as $\widetilde{s}^{l-1}: Y^{l-1} \rightarrow X^{l-1}$ and whose action on each of the remaining terms is the identity.

Continuing this way through each of the factors in the Cartesian product, we conclude that (16) and (17) hold. In particular, $\left.\widetilde{s}\right|_{\mathcal{B}^{-1}(w)}$ has finite fibers.

We ultimately conclude that $\tilde{s}: Y \rightarrow X$ has finite fibers.

The proof of Lemma 5.8, and thus also of Proposition 5.1, is complete.

\section{Computing dynamical degrees}

We now focus our attention on computing the dynamical degrees $\lambda_{k}\left(f_{\rho}\right), 1 \leqslant k \leqslant N$, for all of the generators $f_{\rho}$ of the semi-group $\mathcal{F}^{N}$. The following three facts simplify our task.

(1) Corollary 1.2 establishes that for all $n \geqslant 3$, for all $\rho \in S_{n}$, the map $f_{\rho}: X^{N} \rightarrow X^{N}$ is algebraically stable. As a consequence,

$$
\lambda_{k}\left(f_{\rho}\right)=\text { the spectral radius of }\left(f_{\rho}\right)^{*}: H^{k, k}\left(X^{N} ; \mathbb{C}\right) \rightarrow H^{k, k}\left(X^{N} ; \mathbb{C}\right) .
$$

(2) By Proposition 2.1, since $\widetilde{s}: Y^{N} \rightarrow X^{N}$ has finite fibers, $f^{*}=(g \circ s)^{*}=s^{*} \circ g^{*}$ on all $H^{k, k}\left(X^{N} ; \mathbb{C}\right)$.

(3) Keel's theorem (Theorem 3.4) presents the cohomology ring $H^{*}\left(X^{N} ; \mathbb{C}\right)$ as quotient of the ring generated by all boundary strata by combinatorial relations. 
TABLE 2. Number of strata of codimension $k$ in $X^{N}$.

\begin{tabular}{cccccccc}
\hline & \multicolumn{7}{c}{$k$} \\
\cline { 2 - 8 }$N$ & 0 & 1 & 2 & 3 & 4 & 5 & 6 \\
\hline 0 & 1 & & & & & & \\
1 & 1 & 1 & & & & & \\
2 & 1 & 5 & 1 & & & & \\
3 & 1 & 25 & 105 & 1 & & & \\
4 & 1 & 56 & 490 & 1260 & 1 & & \\
5 & 1 & 119 & 1918 & 9450 & 17325 & 1 & \\
6 & 1 & 246 & 6825 & 63193 & 197774 & 310677 & 1 \\
\hline
\end{tabular}

Point (1) reduces the computation of $\lambda_{k}\left(f_{\rho}\right)$ to the non-dynamical problem of computing

$$
\left(f_{\rho}\right)^{*}: H^{k, k}\left(X^{N} ; \mathbb{C}\right) \rightarrow H^{k, k}\left(X^{N} ; \mathbb{C}\right) .
$$

Point (2) replaces the computation of $\left(f_{\rho}\right)^{*}$ by the computation of

$$
\left(g_{\rho}\right)^{*}: H^{k, k}\left(X^{N} ; \mathbb{C}\right) \rightarrow H^{k, k}\left(X^{N} ; \mathbb{C}\right) \text { and } s^{*}: H^{k, k}\left(X^{N} ; \mathbb{C}\right) \rightarrow H^{k, k}\left(X^{N} ; \mathbb{C}\right) .
$$

This factorization splits the computation into two natural parts: the combinatorial difficulties arising from the permutation $\rho \in S_{n}$ are confined to the automorphism $g_{\rho}: X^{N} \rightarrow X^{N}$, and the difficulties arising from indeterminacy of $f_{\rho}$ are confined to a single map $s: X^{N} \rightarrow X^{N}$.

One major complication is that the number of boundary strata, and the dimensions of the cohomology groups both grow quickly with $N$ as displayed in Tables 1 and 2.

6.1. Pullback under the automorphism $g_{\rho}: X^{N} \rightarrow X^{N}$.

Proposition 6.1. For $\left[D^{S_{1}} \cap \cdots \cap D^{S_{k}}\right] \in H^{k, k}\left(X^{N} ; \mathbb{C}\right)$,

$$
\left(g_{\rho}\right)^{*}\left(\left[D^{S_{1}} \cap \cdots \cap D^{S_{k}}\right]\right)=\left[D^{\rho^{-1}\left(S_{1}\right)} \cap \cdots \cap D^{\rho^{-1}\left(S_{k}\right)}\right] .
$$

Proof. It follows from Proposition 3.11 and the fact that $g_{\rho}$ is unramified that $\left(g_{\rho}\right)^{*}\left(D^{S}\right)=$ $D^{\rho^{-1}(S)}$, as divisors. Thus, on the level of cohomology classes, we have $\left[g_{\rho}^{*} D^{S}\right]=$ $\left[D^{\rho^{-1}(S)}\right]$.

It then follows easily for the codimension $k$ stratum by Corollary 3.5 and taking cup products that

$$
\begin{aligned}
g_{\rho}^{*}\left(\left[D^{S_{1}} \cap \cdots \cap D^{S_{k}}\right]\right) & =\left(g_{\rho}\right)^{*}\left(\left[D^{S_{1}}\right] \smile \cdots \smile\left[D^{S_{k}}\right]\right)=g_{\rho}^{*}\left(\left[D^{S_{1}}\right]\right) \smile \cdots \smile g_{\rho}^{*}\left(\left[D^{S_{k}}\right]\right) \\
& =\left[D^{\rho^{-1}\left(S_{1}\right)}\right] \smile \cdots \smile\left[D^{\rho^{-1}\left(S_{k}\right)}\right]=\left[D^{\rho^{-1}\left(S_{1}\right)} \cap \cdots \cap D^{\rho^{-1}\left(S_{k}\right)}\right] .
\end{aligned}
$$

Note that we are using the fact that $g_{\rho}$ is continuous so that it preserves cup products.

We will construct an explicit basis $\boldsymbol{B}_{k}^{N}$ of $H^{k, k}\left(X^{N} ; \mathbb{C}\right)$ consisting of fundamental cohomology classes of certain codimension $k$ boundary strata. By Proposition $6.1, g_{\rho}^{*}$ induces a permutation on the set of all codimension $k$ boundary strata and Keel's theorem can be used to express $g_{\rho}^{*}\left(\boldsymbol{B}_{k}^{N}\right)$ in terms of $\boldsymbol{B}_{k}^{N}$. The stratified structure of $X^{N}$, coupled 
with the resulting beautifully simple combinatorics of Keel's theorem, makes it possible to directly implement these computations on the computer for all $\rho \in S_{n}$. (Our computations were done in Sage.)

6.2. Pullback action on $H^{1,1}\left(X^{N} ; \mathbb{C}\right)$ under the rational map $s: X^{N} \rightarrow X^{N}$. It will be very helpful for us that $H^{1,1}\left(X^{N} ; \mathbb{C}\right)$ is spanned by the fundamental cohomology classes of the boundary divisors $D^{S}$. This follows immediately from Keel's theorem. However, in order to construct an explicit basis, we recall that for any iterated blow-up $Z$ of projective space, a basis of $H^{1,1}(Z ; \mathbb{C})$ is the fundamental cohomology class of the proper transform of any hyperplane, together with the fundamental cohomology classes of each of the exceptional divisors [GH, p. 605].

Each of the centers of blow-up used in the construction of $X^{N}$ is (the proper transform of) a linear space of the form

$$
0=z_{i_{1}}=\cdots=z_{i_{j}} \quad \text { or } \quad z_{i_{1}}=\cdots=z_{i_{j}} .
$$

In the isomorphism given by Kapranov's theorem (Theorem 3.6), the exceptional divisors over these centers correspond to the boundary divisors $D^{S}$, where

$$
S=\left\{p_{1}, p_{i_{1}+2}, \ldots, p_{i_{j}+2}\right\} \quad \text { or } \quad S=\left\{p_{i_{1}+2}, \ldots, p_{i_{j}+2}\right\}
$$

respectively. Thus, as an ordered basis for $H^{1,1}\left(X^{N} ; \mathbb{C}\right)$ one can take

$$
\boldsymbol{B}_{1}^{N}=\left\{\left[D^{S_{1}}\right], \ldots,\left[D^{S_{\ell}}\right]\right\}
$$

where $S_{1}=\left\{p_{1}, p_{3}\right\}$ corresponds to the proper transform of the hyperplane $z_{1}=0$ and $S_{2}, \ldots, S_{\ell}$ are all subsets of $P$ with $2<\left|S_{i}\right| \leqslant n-2$ and $p_{2} \notin S_{i}$. In particular, $\ell=$ $2^{n-1}-\left(\begin{array}{l}n \\ 2\end{array}\right)-1$. We order the $S_{i}$ so that the $2^{n-2}-n+1$ containing $p_{1}$ are listed before those not containing $p_{1}$.

Let us begin by pulling back $\left[D^{S}\right]$ under $\widetilde{s}^{*}$ for any boundary divisor $D^{S}$, independent of whether it appears in $\boldsymbol{B}_{1}^{N}$. If $\left|S \cap\left\{p_{1}, p_{2}\right\}\right|=1$, then by replacing $S$ with $S^{C}$, if necessary, we have $S=\left\{p_{1}, p_{i_{1}}, \ldots, p_{i_{j}}\right\}$ with $i_{q} \geqslant 3$ for $1 \leqslant q \leqslant j$. Let $\mathcal{D}^{S}$ denote the divisor in $Y^{N}$ obtained as the proper transform of the exceptional divisor obtained by blowing up (the proper transform of) $0=z_{i_{1}}=\cdots=z_{i_{j}}$.

LEMMA 6.2. If $\left|S \cap\left\{p_{1}, p_{2}\right\}\right|=1$, then $\widetilde{s}^{*}\left(\left[D^{S}\right]\right)=2\left[\mathcal{D}^{S}\right]$.

Proof. Notice that the whole construction of $\widetilde{s}: Y^{N} \rightarrow X^{N}$ from $s_{0}: \mathbb{P}^{N} \rightarrow \mathbb{P}^{N}$ that is outlined in diagram (11) commutes with any permutation of the underlying homogeneous coordinates of $\mathbb{P}^{N}$. Therefore, without loss of generality, we can suppose that $S=$ $\{1,3,4, \ldots, j+2\}$ with $D^{S}$ corresponding to the proper transform of $0=z_{1}=\cdots=z_{j}$.

We will use the notation $\left(D^{S}\right)$ when we consider $D^{S}$ as a locally principal divisor with multiplicity. It will be somewhat easier to pull back the divisor $\left(D^{S}\right)$ instead of pulling back the cohomology class $\left[D^{S}\right]$. This will be sufficient for our purposes, because of the following commutative diagram, which is adapted to our setting from [GH, p. 139]:

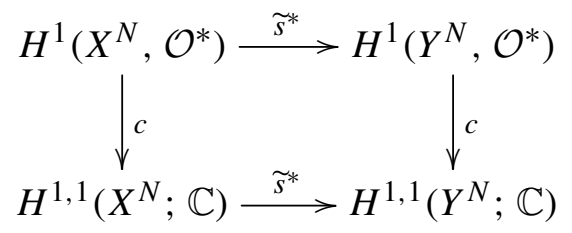


The cohomology groups in the first row describe the linear equivalence classes of locally principal divisors and the vertical arrows denote the Chern class.

Throughout our calculations, we will appeal to $s_{0}: \mathbb{P}^{N} \rightarrow \mathbb{P}^{N}$ which is given by

$$
\left[w_{1}: \cdots: w_{N+1}\right]=\left[z_{1}^{2}: \cdots: z_{N+1}^{2}\right]=s_{0}\left(\left[z_{1}: \cdots: z_{N+1}\right]\right) .
$$

The case $j=1$ is special since $D^{S} \equiv D^{\left\{p_{1}, p_{3}\right\}}$ corresponds to the proper transform of $w_{1}=0$ under all of the blow-ups used to construct $X^{N}$ and $\mathcal{D}^{S}$ corresponds to the proper transform of $z_{1}=0$ under all of the blow-ups used to construct $Y^{N}$. Moreover, it is clear from the commutative diagram (11) that $\widetilde{s}^{-1}\left(D^{S}\right)=\mathcal{D}^{S}$. It remains to keep track of multiplicities. The affine coordinates $v_{1}=\left(w_{1} / w_{N+1}\right), \ldots, v_{N}=\left(w_{N} / w_{N+1}\right)$ serve as local coordinates on $X^{N}$ in a neighborhood of generic points of $D^{S}$ and the affine coordinates $u_{1}=\left(z_{1} / z_{N+1}\right), \ldots, u_{N}=\left(z_{N} / z_{N+1}\right)$ serve as local coordinates on $Y^{N}$ in a neighborhood of generic points of $\mathcal{D}^{S}$. (Here, 'generic' means points which are not on any of the exceptional divisors). Since $\left(D^{S}\right)$ is given locally at generic points by $v_{1}=0$ and $\widetilde{s}\left(u_{1}, \ldots, u_{N}\right)=\left(u_{1}^{2}, \ldots, u_{N}^{2}\right)$, we have that $\widetilde{s}^{*}\left(\left(D^{S}\right)\right)$ is given locally at generic points by $u_{1}^{2}=0$. This gives $\widetilde{s}^{*}\left(\left(D^{S}\right)\right)=2\left(\mathcal{D}^{S}\right)$ and hence $\widetilde{s}^{*}\left(\left[D^{S}\right]\right)=2\left[\mathcal{D}^{S}\right]$, by diagram (19).

The case $j>1$ will be similar, except that we need to describe generic points of $D^{S}$ and $\mathcal{D}^{S}$ using blow-up coordinates. Let us again use the affine coordinates $\left(v_{1}, \ldots, v_{N}\right)$ on $\mathbb{P}^{N} \backslash\left\{w_{N+1}=0\right\}$ and $\left(u_{1}, \ldots, u_{N}\right)$ on $\mathbb{P}^{N} \backslash\left\{z_{N+1}=0\right\}$. At points of the proper transform of $0=v_{1}=\cdots=v_{j}$ not lying on any exceptional divisors resulting from blow-ups of lower-dimensional centers, the blow-up of this center is given by

$$
\left\{\left(v_{1}, \ldots, v_{N}\right) \times\left[n_{1}: \ldots: n_{j}\right] \in \mathbb{C}^{N} \times \mathbb{P}^{j-1} \mid\left(v_{1}, \ldots, v_{j}\right) \sim\left(n_{1}, \ldots, n_{j}\right)\right\} .
$$

Local coordinates on $X^{N}$ in a neighborhood of generic points of $D^{S}$ are given by $\left(\left(n_{1} / n_{j}\right), \ldots,\left(n_{j-1} / n_{j}\right), v_{j}, \ldots, v_{N}\right)$, and in these coordinates $\left(D^{S}\right)$ is given by $v_{j}=0$.

Generic points of $\mathcal{D}^{S}$ can be described by the blow-up of $0=u_{1}=\cdots=u_{j}$, which is given by

$$
\left\{\left(u_{1}, \ldots, u_{N}\right) \times\left[m_{1}: \cdots: m_{j}\right] \in \mathbb{C}^{N} \times \mathbb{P}^{j-1} \mid\left(u_{1}, \ldots, u_{j}\right) \sim\left(m_{1}, \ldots, m_{j}\right)\right\} .
$$

Similarly, local coordinates on $Y^{N}$ in a neighborhood of generic points of $\mathcal{D}^{S}$ are given by $\left(\left(m_{1} / m_{j}\right), \ldots,\left(m_{j-1} / m_{j}\right), u_{j}, \ldots, u_{N}\right)$. In these systems of local coordinates,

$$
\widetilde{s}\left(\frac{m_{1}}{m_{j}}, \ldots, \frac{m_{j-1}}{m_{j}}, u_{j}, \ldots, u_{N}\right)=\left(\left(\frac{m_{1}}{m_{j}}\right)^{2}, \ldots,\left(\frac{m_{j-1}}{m_{j}}\right)^{2}, u_{j}^{2}, \ldots, u_{N}^{2}\right) .
$$

Therefore, at generic points of $\mathcal{D}^{S}, \widetilde{s}^{*}\left(\left(D^{S}\right)\right)$ is given by $u_{j}^{2}=0$. This gives $\widetilde{s}^{*}\left(\left(D^{S}\right)\right)=2\left(\mathcal{D}^{S}\right)$ and hence $\widetilde{s}^{*}\left(\left[D^{S}\right]\right)=2\left[\mathcal{D}^{S}\right]$, by diagram $(19)$.

If $\left|S \cap\left\{p_{1}, p_{2}\right\}\right|=0$ or 2 , then replacing $S$ with $S^{C}$, if necessary, we have $S=$ $\left\{p_{i_{1}}, \ldots, p_{i_{j}}\right\}$ with $i_{q} \neq 1,2$ for $1 \leqslant q \leqslant j$. Let $\mathcal{D}_{ \pm \pm \cdots \pm}^{S}$ denote the divisor in $Y^{N}$ obtained as proper transform of the exceptional divisor obtained by blowing up (the proper transform of)

$$
z_{i_{1}}= \pm z_{i_{2}}=\cdots= \pm z_{i_{j}} .
$$


LEMMA 6.3. If $\left|S \cap\left\{p_{1}, p_{2}\right\}\right|=0$ or 2 , then

$$
\widetilde{s}^{*}\left(\left[D^{S}\right]\right)=\sum\left[\mathcal{D}_{ \pm \pm \cdots \pm}^{S}\right]
$$

where the sum is taken over the $2^{j-1}$ possible choices of signs in (20).

Proof. The proof will be quite similar to that of Lemma 6.2. It will again be simpler to pull back the divisor $\left(D^{S}\right)$ rather than the cohomology class $\left[D^{S}\right]$ and we can again assume, without loss of generality, that $S=\{3,4, \ldots, 2+j\}$.

If $j=2, D^{S} \equiv D^{\left\{p_{3}, p_{4}\right\}}$ is the proper transform of $z_{1}=z_{2}$ under all of the blowups used to construct $X^{N}$ from $\mathbb{P}^{N}$. Similarly, $\mathcal{D}_{ \pm}^{S}$ is the proper transform of $z_{1}= \pm z_{2}$ under all of the blow-ups used to construct $Y^{N}$. The local coordinates $v_{1}, \ldots, v_{N}$ on $\mathbb{P}^{N} \backslash\left\{w_{N+1}=0\right\}$ from Lemma 6.2 serve as local coordinates on $X^{N}$ in a neighborhood of generic points of $D^{S}$. Meanwhile, the local coordinates $u_{1}, \ldots, u_{N}$ in $\mathbb{P}^{N} \backslash\left\{z_{N+1}=0\right\}$ serve as local coordinates on $Y^{N}$ in a neighborhood of generic points in a neighborhood of $\mathcal{D}_{ \pm}^{S}$. Moreover, it is clear from the commutative diagram (11) that $\widetilde{s}^{-1}\left(D^{S}\right)=\mathcal{D}_{+}^{S} \cup \mathcal{D}_{-}^{S}$. Thus, it remains to keep track of multiplicities. The divisor $\left(D^{S}\right)$ is locally given at generic points by $v_{1}-v_{2}=0$. At points of $Y^{N}$ where $u_{1}, \ldots, u_{N}$ serve as coordinates and at points of $X^{N}$ where $v_{1}, \ldots, v_{N}$ serve as coordinates, the map $\widetilde{s}$ is given by $\widetilde{s}\left(u_{1}, \ldots, u_{N}\right)=\left(u_{1}^{2}, \ldots, u_{N}^{2}\right)$. Since $\left(D^{S}\right)$ is locally given by $v_{1}-v_{2}=0$, it follows that $\widetilde{s}^{*}\left(\left(D^{S}\right)\right)$ is given at generic points by $u_{1}^{2}-u_{2}^{2}=\left(u_{1}-u_{2}\right)\left(u_{1}+u_{2}\right)$. Since the first factor describes $\left(\mathcal{D}_{+}^{S}\right)$ and the second factor describes $\left(\mathcal{D}_{-}^{S}\right)$, we conclude that $\widetilde{s}^{*}\left(\left(D^{S}\right)\right)=$ $\left(\mathcal{D}_{+}^{S}\right)+\left(\mathcal{D}_{-}^{S}\right)$. By commutative diagram (19), this implies that $\widetilde{s}^{*}\left(\left[D^{S}\right]\right)=\left[\mathcal{D}_{+}^{S}\right]+\left[\mathcal{D}_{-}^{S}\right]$.

The case $j>2$ will be similar, except that we will need to use blow-up coordinates. It is clear from the commutative diagram (11) that

$$
\widetilde{s}^{-1}\left(D^{S}\right)=\bigcup \mathcal{D}_{ \pm \ldots \pm}^{S}
$$

Thus, it remains to compute the multiplicity of each contribution. Let us again use the affine coordinates $\left(v_{1}, \ldots, v_{N}\right)$ on $\mathbb{P}^{N} \backslash\left\{w_{N+1}=0\right\}$ and $\left(u_{1}, \ldots, u_{N}\right)$ on $\mathbb{P}^{N} \backslash\left\{z_{N+1}=0\right\}$. At points of the proper transform of $v_{1}=\cdots=v_{j}$ not lying on any exceptional divisors resulting from blow-ups of lower-dimensional centers, the blow-up of this center is given by

$$
\begin{aligned}
& \left\{\left(v_{1}, \ldots, v_{N}\right) \times\left[n_{1}: \cdots: n_{j-1}\right]\right. \\
& \left.\quad \in \mathbb{C}^{N} \times \mathbb{P}^{j-2} \mid\left(v_{1}-v_{j}, \ldots, v_{j-1}-v_{j}\right) \sim\left(n_{1}, \ldots, n_{j-1}\right)\right\} .
\end{aligned}
$$

Local coordinates on $X^{N}$ in a neighborhood of generic points of $D^{S}$ are given by

$$
\left(\frac{n_{1}}{n_{j-1}}, \ldots, \frac{n_{j-2}}{n_{j-1}}, v_{j-1}-v_{j}, v_{j}, \ldots, v_{N}\right) .
$$

Generic points of $\mathcal{D}_{ \pm \pm \cdots \pm}^{S}$ can be described by the blow-up of $z_{1}= \pm z_{2}= \pm z_{j}$, which is given by

$$
\begin{aligned}
& \left\{\left(u_{1}, \ldots, u_{N}\right) \times\left[m_{1}: \cdots: m_{j-1}\right]\right. \\
& \left.\quad \in \mathbb{C}^{N} \times \mathbb{P}^{j-2} \mid\left(v_{1} \mp v_{j}, \ldots, v_{j-1} \mp v_{j}\right) \sim\left(m_{1}, \ldots, m_{j-1}\right)\right\} .
\end{aligned}
$$


Local coordinates on $Y^{N}$ in a neighborhood of generic points of $\mathcal{D}_{ \pm \pm \cdots \pm}^{S}$ are given by

$$
\left(\frac{m_{1}}{m_{j-1}}, \ldots, \frac{m_{j-2}}{m_{j-1}}, u_{j-1} \mp u_{j}, u_{j}, \ldots, u_{N}\right) \text {. }
$$

In these local coordinates,

$$
\begin{aligned}
& \widetilde{s}\left(\frac{m_{1}}{m_{j-1}}, \ldots, \frac{m_{j-2}}{m_{j-1}}, u_{j-1} \mp u_{j}, u_{j}, \ldots, u_{N}\right) \\
& \quad=\left(\frac{m_{1}}{m_{j-1}}, \ldots, \frac{m_{j-2}}{m_{j-1}}, u_{j-1}^{2} \mp u_{j}^{2}, u_{j}^{2}, \ldots, u_{N}^{2}\right) .
\end{aligned}
$$

Since $\left(D^{S}\right)$ is given by $v_{j-1}-v_{j}=0$, in these coordinates $\widetilde{s}^{*}\left(\left(D^{S}\right)\right)$ is given by $u_{j-1}^{2} \mp u_{j}^{2}=\left(u_{j-1}-u_{j}\right)\left(u_{j-1}+u_{j}\right)$. Since $\left(\mathcal{D}_{ \pm \pm \cdots \pm}^{S}\right)$ is given locally by exactly one of these two linear factors, we see that for each combination of \pm , the preimage $\mathcal{D}_{ \pm \pm \cdots \pm}^{S}$ is counted with multiplicity one. Thus,

$$
\widetilde{s}^{*}\left(\left(D^{S_{i}}\right)\right)=\sum\left(\mathcal{D}_{ \pm \pm \cdots \pm}^{S_{i}}\right)
$$

By commutative diagram (19) this gives (21).

Remark 6.4. We will refer to divisors $D^{S}$ with $\left|S \cap\left\{p_{1}, p_{2}\right\}\right|=1$ as ramified divisors and those with $\left|S \cap\left\{p_{1}, p_{2}\right\}\right|=0$ or 2 as unramified divisors.

We now return to our basis

$$
\boldsymbol{B}_{1}^{N}=\left\{\left[D^{S_{1}}\right], \ldots,\left[D^{S_{\ell}}\right]\right\}
$$

where $S_{1}=\left\{p_{1}, p_{3}\right\}$ corresponds to the proper transform of the hyperplane $z_{1}=0$ and $S_{2}, \ldots, S_{\ell}$ are all subsets of $P$ with $2<\left|S_{i}\right| \leqslant n-2$ and $p_{2} \notin S_{i}$.

PROPOSITION 6.5. With respect to the ordered basis $\boldsymbol{B}_{1}^{N}$,

$$
s^{*}: H^{1,1}\left(X^{N} ; \mathbb{C}\right) \rightarrow H^{1,1}\left(X^{N} ; \mathbb{C}\right) \quad \text { is given by } s^{*}=\operatorname{diag}(2, \ldots, 2,1, \ldots, 1),
$$

where the first $2^{n-2}-n+1$ entries of the diagonal are 2, corresponding to the ramified divisors $D^{S}$ (those with $\left|S \cap\left\{p_{1}, p_{2}\right\}\right|=1$ ), and the remaining entries are 1, corresponding to the unramified divisors $D^{S}$ (those with $\left|S \cap\left\{p_{1}, p_{2}\right\}\right|=0$ ).

Proof. For any $\left[D_{i}^{S}\right]$ we compute $s^{*}\left(\left[D_{i}^{S}\right]\right)=\operatorname{pr}_{*}\left(\widetilde{s}^{*}\left(\left[D_{i}^{S}\right]\right)\right)$. We will use Lemmas 6.2 and 6.3 to compute $\widetilde{s}^{*}\left(\left[D_{i}^{S}\right]\right)$. We will then use Lemma 2.3 to determine the effect of $\mathrm{pr}_{*}$ on each of the fundamental classes in $\widetilde{s}^{*}\left(\left[D_{i}^{S}\right]\right)$.

Since $\operatorname{pr}: Y^{N} \rightarrow X^{N}$ is a birational morphism, it follows from Zariski's main theorem [H, Ch. III, Corollaries 11.4] that the fibers of pr are connected. In particular, for any irreducible subvariety $V \subseteq Y^{N}$ we will have either $\operatorname{dim}(\operatorname{pr}(V))<\operatorname{dim}(V)$ or $\operatorname{deg}_{\text {top }}\left(\left.\operatorname{pr}\right|_{V}\right)=1$.

First, suppose that $S_{i}=\left\{p_{1}, p_{i_{1}}, \ldots, p_{i_{j}}\right\}$ with $i_{q} \neq 2$ for $1 \leqslant q \leqslant j$. According to Lemma 6.2 we have $\tilde{s}^{*}\left(\left[D^{S_{i}}\right]\right)=2\left[\mathcal{D}^{S_{i}}\right]$. The homogeneous coordinates $\left[z_{1}: \cdots: z_{N+1}\right]$ serve as coordinates on generic points of $Y^{N}$ and the homogeneous coordinates $\left[w_{1}: \cdots: w_{N+1}\right]$ serve as coordinates on generic points of $X^{N}$. It follows from 
commutativity of (7) that in these coordinates $\operatorname{pr}\left(\left[z_{1}: \cdots: z_{N+1}\right]\right)=\left[z_{1}: \cdots: z_{N+1}\right]$. Since the proper transform of $0=z_{i_{i}+2}=z_{i_{j}+2}$ is blown up to construct $Y^{N}$, corresponding to $\mathcal{D}^{S_{i}}$, and the proper transform of $0=w_{i_{i}+2}=w_{i_{j}+2}$ is blown up in the construction of $X^{N}$, corresponding to $D^{S_{i}}$, it follows that pr maps $\mathcal{D}^{S_{i}}$ onto $D^{S_{i}}$. Therefore,

$$
s^{*}\left(\left[D^{S_{i}}\right]\right)=\operatorname{pr}_{*}\left(\widetilde{s}^{*}\left(\left[D^{S_{i}}\right]\right)\right)=\operatorname{pr}_{*}\left(2\left[\mathcal{D}^{S_{i}}\right]\right)=2\left[D^{S_{i}}\right] .
$$

Now suppose that $S_{i}=\left\{p_{i_{1}}, \ldots, p_{i_{j}}\right\}$ with $i_{q} \neq 1,2$ for $1 \leqslant q \leqslant j$. According to Lemma 6.3,

$$
\widetilde{s}^{*}\left(\left[D^{S_{i}}\right]\right)=\sum\left[\mathcal{D}_{ \pm \cdots \pm}^{S_{i}}\right]
$$

As in the previous paragraph, $\operatorname{pr}\left(\mathcal{D}_{+\cdots+}^{S_{i}}\right)=D^{S_{i}}$, implying that $\operatorname{pr}_{*}\left(\left[\mathcal{D}_{+\cdots+}^{S_{i}}\right]\right)=\left[D^{S_{i}}\right]$. Now consider the case that not all of the signs indexing $\mathcal{D}_{ \pm \cdots \pm}^{S_{i}}$ are ' + '. First, notice that since $\mathcal{D}_{ \pm \cdots \pm}^{S_{i}}$ is irreducible, so is $\operatorname{pr}\left(\mathcal{D}_{ \pm \ldots \pm}^{S_{i}}\right)$. By commutativity of (7), we have that $\operatorname{pr}\left(\mathcal{D}_{ \pm \ldots \pm}^{S_{i}}\right)$ lies within

$$
\mathcal{A}^{-1}\left(z_{i_{1}-2}= \pm z_{i_{2}-2}= \pm z_{i_{j}-2}\right)
$$

where $\mathcal{A}: X^{N} \rightarrow \mathbb{P}^{N}$ is the composition of all of the blow-ups used to construct $X^{N}$. Moreover, $\operatorname{pr}\left(\mathcal{D}_{ \pm \cdots \pm}^{S_{i}}\right)$ contains at least one point in each $\mathcal{A}$-fiber over $z_{i_{1}-2}= \pm z_{i_{2}-2}=$ $\pm z_{i_{j}-2}$. However, since at least one of the \pm is minus, generic points of this linear subspace are not on any of the centers of blow-up. Thus, there are points of $\operatorname{pr}\left(\mathcal{D}_{ \pm \ldots \pm}^{S_{i}}\right)$ which have a neighborhood in $\operatorname{pr}\left(\mathcal{D}_{ \pm}^{S_{i} \ldots \pm}\right)$ that is contained within an analytic set of dimension less than $N-1$. Since $\operatorname{pr}\left(\mathcal{D}_{ \pm \cdots \pm}^{S_{i}}\right)$ is irreducible, this implies that $\operatorname{dim}\left(\operatorname{pr}\left(\mathcal{D}_{ \pm \cdots \pm}^{S_{i}}\right)\right)<N-1$. Therefore, if not all of the signs are + , we have $\operatorname{pr}_{*}\left(\left[\mathcal{D}_{ \pm \cdots \pm}^{S_{i}}\right]\right)=0$. We conclude that

$$
s^{*}\left(\left[D^{S_{i}}\right]\right)=\operatorname{pr}_{*}\left(\widetilde{s}^{*}\left(\left[D^{S_{i}}\right]\right)\right)=\operatorname{pr}_{*}\left(\sum\left[\mathcal{D}_{ \pm \cdots \pm}^{S_{i}}\right]\right)=\operatorname{pr}_{*}\left(\left[\mathcal{D}_{+\cdots+}^{S_{i}}\right]\right)=\left[D^{S_{i}}\right] .
$$

6.3. Pullback action on $H^{2,2}\left(X^{3} ; \mathbb{C}\right)$ under the rational map $s: X^{3} \rightarrow X^{3}$. For any projective manifold $X$ and any dominant rational map $f: X \rightarrow X$, it can be quite subtle to keep track of inverse images of subvarieties $V \subseteq X$ of codimension at least 2 , since they may lie in the indeterminacy locus $I_{f}$. For this reason, one must compute preimages (set-theoretic and cohomological) using a resolution of singularities as in (3).

This is even more subtle for the map $s: X^{N} \rightarrow X^{N}$ because pr: $Y^{N} \rightarrow X^{N}$ is defined implicitly by a universal property. Because of these challenges and the computational complexity arising from the dimensions of the $H^{k, k}\left(X^{N} ; \mathbb{C}\right)$ growing exponentially with $N$, we will limit ourselves in this section to $N=3$. The case for $N=3$ and $k=1$ has already been analyzed in $\S 6.2$, so we will focus on $k=2$. With enough computational power and careful book-keeping about preimages lying in the indeterminacy locus, we expect these techniques to extend to arbitrary $N$ and $k$.

One further challenge is that we do not know if the action can be expressed by a diagonal matrix.

Question 1. For any $N$ and any $k \geqslant 2$, does there exist an ordered basis for $H^{k, k}(X ; \mathbb{C})$ consisting of fundamental classes of boundary strata, in which the action of $s^{*}$ is expressed by a diagonal matrix? (Compare to Proposition 6.7, below.) 
The following proposition is stated for general $k$ and $N$.

Proposition 6.6. Let $Z:=D^{S_{1}} \cap \cdots \cap D^{S_{k}} \subseteq X^{N}$ be a codimension $k$ boundary stratum and let $W_{1}, \ldots, W_{\ell} \subseteq X^{N}$ be the irreducible components of $s^{-1}(Z):=$ $\operatorname{pr}\left(\widetilde{s}^{-1}(Z)\right)$ that have codimension exactly $k$. We have

$$
s^{*}[Z]=\sum_{m=1}^{\ell} 2^{r}\left[W_{m}\right]
$$

where $r$ is the number of the boundary divisors among $\left\{D^{S_{1}}, \ldots, D^{S_{k}}\right\}$ that are ramified, that is, those satisfying $\left|S_{i} \cap\left\{p_{1}, p_{2}\right\}\right|=1$.

Some comments are in order.

(1) Since $\widetilde{s}$ has finite fibers, every irreducible component of $s^{-1}(Z)$ has codimension at least $k$. We ignore any preimages of codimension greater than $k$, even those lying entirely in $I_{s} \subseteq X^{N}$.

(2) By Lemma 3.3, $Z$ is uniquely represented as an intersection of boundary divisors, so that the number $r$ is well defined.

Proof. Recall that $s^{*}([Z]):=\operatorname{pr}_{*}\left(\widetilde{s}^{*}([Z])\right)$. Without loss of generality, we can suppose that the first $r$ divisors are ramified and the remaining ones are unramified. From Lemmas 6.2 and 6.3,

$$
\begin{aligned}
\widetilde{s}^{*}([Z]) & =\widetilde{s}^{*}\left(\left[D^{S_{1}}\right] \smile \cdots \smile\left[D^{S_{k}}\right]\right)=\widetilde{s}^{*}\left(\left[D^{S_{1}}\right]\right) \smile \cdots \smile \widetilde{s}^{*}\left(\left[D^{S_{k}}\right]\right) \\
& =2\left[\mathcal{D}^{S_{1}}\right] \smile \cdots \smile 2\left[\mathcal{D}^{S_{r}}\right] \smile\left(\sum\left[\mathcal{D}_{ \pm \pm \cdots \pm}^{S_{r+1}}\right]\right) \smile \cdots \smile\left(\sum\left[\mathcal{D}_{ \pm \pm \cdots \pm}^{S_{k}}\right]\right) \\
& =2^{r} \sum_{m=1}^{j}\left[V_{m}\right]
\end{aligned}
$$

where each $V_{m}$ is an irreducible component of $\widetilde{s}^{-1}\left(D^{S_{1}}\right) \cap \cdots \cap \widetilde{s}^{-1}\left(D^{S_{k}}\right)$. Each of these components has codimension $k$ since $\tilde{s}$ has finite fibers. Notice that each $k$-fold iterated cup product obtained when expanding the sum corresponds to $k$ fundamental classes of divisors intersecting transversally. This is why the fundamental cohomology class of each component $V_{m}$ does not get an extra multiplicity.

According to Lemma 2.3, any $V_{m}$ with $\operatorname{dim}\left(\operatorname{pr}\left(V_{m}\right)\right)<\operatorname{dim}\left(V_{m}\right)$ will have $\operatorname{pr}_{*}\left(\left[V_{m}\right]\right)=0$. Removing any such $V_{m}$ from our list (and reordering if necessary), we can assume that the first $\ell$ components $V_{1}, \ldots, V_{\ell}$ are mapped by pr onto $W_{1}, \ldots, W_{\ell}$ of the same dimension and the remaining components are decreased in dimension by the map pr. It follows from Zariski's main theorem [H, Ch. III, Corollaries 11.4] that $\operatorname{deg}_{\text {top }}\left(\left.\mathrm{pr}\right|_{V_{m}}\right)=1$ for $1 \leqslant m \leqslant \ell$. In conclusion,

$$
s^{*}([Z])=\operatorname{pr}_{*}\left(\widetilde{s}^{*}([Z])\right)=\operatorname{pr}_{*}\left(2^{r} \sum_{m=1}^{j}\left[V_{m}\right]\right)=2^{r} \sum_{m=1}^{\ell}\left[W_{m}\right] .
$$


We will construct an ordered basis $\boldsymbol{B}_{2}^{3}$ for $H^{2,2}\left(X^{3}, \mathbb{C}\right)$ using intersections of the boundary divisors indexed by the following subsets of $P=\left\{p_{1}, \ldots, p_{6}\right\}$ :

$$
\begin{array}{llll}
S_{1}:=\left\{p_{1}, p_{3}, p_{4}, p_{5}\right\}, & S_{6}:=\left\{p_{1}, p_{3}, p_{4}\right\}, & S_{11}:=\left\{p_{1}, p_{5}, p_{6}\right\}, & S_{16}:=\left\{p_{1}, p_{2}, p_{6}\right\}, \\
S_{2}:=\left\{p_{1}, p_{3}, p_{4}, p_{6}\right\}, & S_{7}:=\left\{p_{1}, p_{3}, p_{5}\right\}, & S_{12}:=\left\{p_{1}, p_{2}\right\}, & S_{17}:=\left\{p_{1}, p_{4}\right\}, \\
S_{3}:=\left\{p_{1}, p_{3}, p_{5}, p_{6}\right\}, & S_{8}:=\left\{p_{1}, p_{3}, p_{6}\right\}, & S_{13}:=\left\{p_{1}, p_{2}, p_{3}\right\}, & S_{18}:=\left\{p_{3}, p_{4}\right\} . \\
S_{4}:=\left\{p_{1}, p_{4}, p_{5}, p_{6}\right\}, & S_{9}:=\left\{p_{1}, p_{4}, p_{5}\right\}, & S_{14}:=\left\{p_{1}, p_{2}, p_{4}\right\}, & \\
S_{5}:=\left\{p_{1}, p_{3}\right\}, & S_{10}:=\left\{p_{1}, p_{4}, p_{6}\right\}, & S_{15}:=\left\{p_{1}, p_{2}, p_{5}\right\},
\end{array}
$$

By Keel's theorem, $\operatorname{dim}\left(H^{2,2}\left(X^{3} ; \mathbb{C}\right)\right)=16$. We will use as many ramified codimension-two boundary strata as possible in order to make the expression of $s^{*}$ in $\boldsymbol{B}_{2}^{3}$ as close to being diagonal as possible. Our first 11 strata are obtained as intersections of two ramified divisors:

$$
\begin{array}{llll}
Z_{1}:=D^{S_{1}} \cap D^{S_{5}}, & Z_{4}:=D^{S_{2}} \cap D^{S_{5}}, & Z_{7}:=D^{S_{2}} \cap D^{S_{10}}, & Z_{10}:=D^{S_{4}} \cap D^{S_{17}}, \\
Z_{2}:=D^{S_{1}} \cap D^{S_{7}}, & Z_{5}:=D^{S_{2}} \cap D^{S_{6}}, & Z_{8}:=D^{S_{3}} \cap D^{S_{5}}, & Z_{11}:=D^{S_{5}} \cap D^{S_{7}} . \\
Z_{3}:=D^{S_{1}} \cap D^{S_{9}}, & Z_{6}:=D^{S_{2}} \cap D^{S_{8}}, & Z_{9}:=D^{S_{3}} \cap D^{S_{11}}, &
\end{array}
$$

Let $Z=D^{S_{i}} \cap D^{S_{j}}$ be any one of these 11 boundary strata. Since each is ramified we have $\widetilde{s}^{-1}\left(D^{S_{i}}\right)=\mathcal{D}^{S_{i}}$ and $\widetilde{s}^{-1}\left(D^{S_{j}}\right)=\mathcal{D}^{S_{j}}$. Hence, $\widetilde{s}^{-1}(Z)=\mathcal{D}^{S_{i}} \cap \mathcal{D}^{S_{j}}$. Similarly to the proof of Proposition 6.5, we have $\operatorname{pr}\left(\mathcal{D}^{S_{i}} \cap \mathcal{D}^{S_{j}}\right)=Z$. Since both $D^{S_{i}}$ and $D^{S_{j}}$ are ramified, it follows from Proposition 6.6 that $s^{*}([Z])=2^{2}[Z]$. In summary,

$$
s^{*}\left(\left[Z_{i}\right]\right)=2^{2}\left[Z_{i}\right] \text { for all } 1 \leqslant i \leqslant 11 .
$$

There are four more ramified strata of codimension two we will use for our basis:

$$
\begin{gathered}
Z_{12}:=D^{S_{1}} \cap D^{S_{16}}, \quad Z_{13}:=D^{S_{2}} \cap D^{S_{15}}, \\
Z_{14}:=D^{S_{3}} \cap D^{S_{14}}, \quad \text { and } \quad Z_{15}:=D^{S_{4}} \cap D^{S_{13}} .
\end{gathered}
$$

For each of them, the first term in the intersection is ramified and the second one is unramified.

First consider $Z_{12}=D^{S_{1}} \cap D^{S_{16}}$. Recall that we use the normalization

$$
\begin{gathered}
\varphi\left(p_{1}\right)=0, \quad \varphi\left(p_{2}\right)=\infty, \quad \varphi\left(p_{3}\right)=z_{1}, \quad \varphi\left(p_{4}\right)=z_{2}, \\
\varphi\left(p_{5}\right)=z_{3}, \quad \text { and } \varphi\left(p_{6}\right)=z_{4} .
\end{gathered}
$$

With respect to the coordinates obtained from this normalization,

$$
D^{S_{1}}=\mathcal{A}^{-1}([0: 0: 0: 1]) \cong X^{2}
$$

and $\mathcal{D}^{S_{1}}=\widetilde{s}^{-1}\left(D^{S_{1}}\right)=\mathcal{B}^{-1}([0: 0: 0: 1]) \cong Y^{2}$. As in the proof of Lemma 5.8, under these identifications of the fibers with $X^{2}$ and $Y^{2}$,

$$
\left.\widetilde{s}\right|_{\mathcal{B}^{-1}([0: 0: 0: 1])}: \mathcal{B}^{-1}([0: 0: 0: 1]) \rightarrow \mathcal{A}^{-1}([0: 0: 0: 1])
$$

is $\widetilde{s}^{2}: Y^{2} \rightarrow X^{2}$ and

$$
\left.\operatorname{pr}\right|_{\mathcal{B}} ^{-1}([0: 0: 0: 1]): \mathcal{B}^{-1}([0: 0: 0: 1]) \rightarrow \mathcal{A}^{-1}([0: 0: 0: 1])
$$

is $\operatorname{pr}^{2}: Y^{2} \rightarrow X^{2}$. (We are using superscripts on the names of the maps to denote the dimensions of the domain/codomain, as in Lemma 5.8.) Therefore, computing 
$s^{-1}\left(Z_{12}\right):=\operatorname{pr}\left(\widetilde{s}^{-1}\left(Z_{12}\right)\right)$ amounts to computing $\left(s^{2}\right)^{-1}(W):=\operatorname{pr}^{2}\left(\left(\widetilde{s}^{2}\right)^{-1}(W)\right)$, where $W$ is the divisor in $X^{2}$ obtained from intersecting $\mathcal{A}^{-1}([0: 0: 0: 1]) \cong X^{2}$ with $D^{S_{16}}$. Recall that $X^{2}$ is the blow-up of $\mathbb{P}^{2}$ at $[0: 0: 1],[0: 1: 0],[1: 0: 0]$, and $[1: 1: 1]$, where in this context $\mathbb{P}^{2}$ is the exceptional divisor over $[0: 0: 0: 1]$ obtained in the first round of blow-ups of $\mathbb{P}^{3}$ used to construct $X^{3}$. Since $D^{S_{16}}$ is obtained by blowing up the proper transform of $z_{1}=z_{2}=z_{3}$, the intersection $W$ corresponds in $\mathcal{A}^{-1}([0: 0: 0: 1])$ to the blow-up of $\mathbb{P}^{2}$ at $[1: 1: 1]$. Therefore, the preimages under $\widetilde{s}^{2}$ will correspond in $\mathcal{B}^{-1}([0: 0: 0: 1])$ to the blow-ups of $\mathbb{P}^{2}$ at $[1: \pm 1: \pm 1]$. As in the proof of Proposition 6.5 , $\mathrm{pr}^{2}$ will crush each of these blow-ups other than the one at $[1: 1: 1]$. Therefore, the only component of $\left(s^{2}\right)^{-1}(W):=\operatorname{pr}^{2}\left(\left(\widetilde{s}^{2}\right)^{-1}(W)\right)$ of dimension one is the blow-up of $\mathbb{P}^{2}$ at [1:1:1]. Considered in $X^{3}$, this is just $Z_{12}$.

Essentially the same proof shows that for $13 \leqslant i \leqslant 15$, the only component of $s^{-1}\left(Z_{i}\right)$ having dimension one is $Z_{i}$. Proposition 6.6 gives that

$$
s^{*}\left(\left[Z_{i}\right]\right)=2\left[Z_{i}\right] \text { for all } 12 \leqslant i \leqslant 15 .
$$

We require one more basis element, which unfortunately will not pull back to a multiple of itself. Let

$$
Z_{16}:=D^{S_{12}} \cap D^{S_{18}}
$$

It can be readily verified from Theorem 3.4 that the ordered set

$$
\boldsymbol{B}_{2}^{3}=\left\{\left[Z_{1}\right], \ldots,\left[Z_{16}\right]\right\}
$$

is a basis of $H^{2,2}\left(X^{3} ; \mathbb{C}\right)$.

We will again need to use coordinates from the blow-up description of $X^{3}$ given in $\$ 3.4$ to compute $s^{*}\left(\left[Z_{16}\right]\right)$. To simplify notation, let us write $Z \equiv Z_{16}$. Since neither $D^{S_{12}}$ nor $D^{S_{18}}$ is ramified, Proposition 6.6 gives that $s^{*}[Z]$ will be the sum of fundamental classes of the components of $s^{-1}(Z)$ of dimension one, each with multiplicity one.

Recall that we use the normalization stated in (23). In these coordinates, $Z$ is the intersection of $E_{[1: 1: 1: 1]}$ with the proper transform of the hyperplane $z_{1}=z_{2}$. Consider the preimages $[1: \pm 1: \pm 1: \pm 1] \in s_{0}^{-1}([1: 1: 1: 1])$. Because these points are not critical, there are neighborhoods $\mathcal{B}^{-1}(U)$ and $\mathcal{A}^{-1}(V)$ of $\mathcal{B}^{-1}([1: \pm 1: \pm 1: \pm 1])$ and $\mathcal{A}^{-1}([1: 1: 1: 1])$, respectively, so that

$$
\left.\widetilde{s}\right|_{\mathcal{B}^{-1}(U)}: \mathcal{B}^{-1}(U) \rightarrow \mathcal{A}^{-1}(V)
$$

is a biholomorphism (see the proof of Lemma 5.8). In particular, there are eight irreducible preimages $z_{ \pm, \pm, \pm}$of $Z$ under $\widetilde{s}$, with one of them in each such neighborhood $\mathcal{B}^{-1}(U)$. We must determine which of them have image under the map pr of dimension one.

There is a sufficiently small neighborhood $U$ of $[1: 1: 1: 1]$ so that pr maps $\mathcal{B}^{-1}(U)$ biholomorphically onto $\mathcal{A}^{-1}(U)$. In particular, $\operatorname{pr}\left(Z_{+,+,+}\right)=Z$, so that $[Z]$ contributes to $s^{*}([Z])$.

Now consider the three components indexed by two minus signs. Since the points $[1: \pm 1: \pm 1: \pm 1]$ with exactly two minus signs are not on $A_{0} \cup A_{1}$, for these points we 
have that $\mathcal{A}^{-1}([1: \pm 1: \pm 1: \pm 1])$ is a single point. Commutativity of the diagram

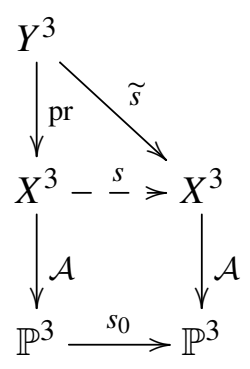

implies that $\operatorname{pr}\left(Z_{ \pm, \pm, \pm}\right) \subseteq \mathcal{A}^{-1}([1: \pm 1: \pm 1: \pm 1])$. Therefore, the fundamental classes of these preimages do not contribute to $s^{*}([Z])$.

The remaining four components $z_{-1,1,1}, z_{1,-1,1}, z_{1,1,-1}$, and $z_{-1,-1,-1}$ require a more careful analysis because the corresponding points in $\mathbb{P}^{3}$ satisfy

$$
[1:-1: 1: 1],[1: 1:-1: 1],[1: 1: 1:-1],[1:-1:-1:-1] \in A^{1},
$$

resulting in

$$
\left.\operatorname{pr}\right|_{\mathcal{B}^{-1}([1: \pm 1: \pm 1: \pm 1])}: \mathcal{B}^{-1}([1: \pm 1: \pm 1: \pm 1]) \rightarrow \mathcal{A}^{-1}([1: \pm 1: \pm 1: \pm 1])
$$

being a map from the two-dimensional manifold $\mathcal{B}^{-1}([1: \pm 1: \pm 1: \pm 1]) \cong X^{2}$ to the onedimensional manifold $\mathcal{A}^{-1}([1: \pm 1: \pm 1: \pm 1]) \cong X^{1}$.

Consider $\left.\widetilde{s}\right|_{\mathcal{B}^{-1}([1:-1: 1: 1])}: \mathcal{B}^{-1}([1:-1: 1: 1]) \rightarrow \mathcal{A}^{-1}([1: 1: 1: 1])$. The fiber $\mathcal{B}^{-1}([1:-1: 1: 1])$ is obtained by first blowing up the point $[1:-1: 1: 1]$ and then blowing up the proper transforms of the lines

$$
z_{1}=-z_{2}=z_{3}, \quad z_{1}=-z_{2}=z_{4}, \quad z_{1}=z_{3}=z_{4}, \quad-z_{2}=z_{3}=z_{4} .
$$

Similarly, the fiber $\mathcal{A}^{-1}([1: 1: 1: 1])$ is obtained by first blowing up the point $[1: 1: 1: 1]$ and then blowing up the proper transforms of the lines

$$
z_{1}=z_{2}=z_{3}, \quad z_{1}=z_{2}=z_{4}, \quad z_{1}=z_{3}=z_{4}, \quad z_{2}=z_{3}=z_{4} .
$$

Consider just the point blow-ups at $[1:-1: 1: 1]$ and $[1: 1: 1: 1]$, respectively. There are coordinates

$\left(\left[y_{1}: y_{2}: y_{3}: y_{4}\right],\left[m_{1}: m_{2}: m_{3}\right]\right) \quad$ where $\left(m_{1}, m_{2}, m_{3}\right) \sim\left(y_{1}-y_{4}, y_{2}+y_{4}, y_{3}-y_{4}\right)$, and

$$
\left(\left[x_{1}: x_{2}: x_{3}: x_{4}\right],\left[n_{1}: n_{2}: n_{3}\right]\right) \quad \text { where }\left(n_{1}, n_{2}, n_{3}\right) \sim\left(x_{1}-x_{4}, x_{2}-x_{4}, x_{3}-x_{4}\right)
$$

in neighborhoods of $E_{[1:-1: 1: 1]}$ within $Y_{1}^{3}$ and of $E_{[1: 1: 1: 1]}$ within $X_{1}^{3}$. In fact, these serve as coordinates in a neighborhood of the generic points of $\mathcal{B}^{-1}([1:-1: 1: 1])$ and $\mathcal{A}^{-1}\left([1: 1: 1: 1]\right.$ ), within $Y^{3}$ and $X^{3}$, respectively. (By 'generic', we mean points that are not altered by the blow-ups of the proper transforms of the lines (24) and (25).)

In these coordinates, $\left.\widetilde{s}\right|_{\mathcal{B}^{-1}([1:-1: 1: 1])}: \mathcal{B}^{-1}([1:-1: 1: 1]) \rightarrow \mathcal{A}^{-1}([1: 1: 1: 1])$ is given by

$$
\left.\widetilde{s}\right|_{\mathcal{B}^{-1}([1:-1: 1: 1])}\left(\left[m_{1}: m_{2}: m_{3}\right]\right)=\left[m_{1}: m_{2}: m_{3}\right]
$$


At generic points of $\mathcal{A}^{-1}([1: 1: 1: 1]), Z$ is described by the equation $n_{1}=n_{2}$. Therefore, $z_{-,+,+}=\left(\left.\widetilde{s}\right|_{\mathcal{B}^{-1}([1:-1: 1: 1])}\right)^{-1}(Z)$ is described at generic points of $\mathcal{B}^{-1}([1:-1: 1: 1])$ by

$$
m_{1}=m_{2} \text {. }
$$

The fiber $\mathcal{A}^{-1}([1:-1: 1: 1])$ is a result of blowing up the line $\left\{z_{1}=z_{3}=z_{4}\right\} \in A^{1}$. Coordinates in a neighborhood of this fiber are given by

$$
\left(\left[z_{1}: z_{2}: z_{3}: z_{4}\right],\left[p_{1}: p_{2}\right]\right) \quad \text { where }\left(p_{1}, p_{2}\right) \sim\left(z_{1}-z_{4}, z_{3}-z_{4}\right) .
$$

Notice that generic points of $Y^{3}$ (i.e. those not in $\mathcal{B}^{-1}\left(B^{0} \cup B^{1}\right)$ ) can be described by the homogeneous coordinates $\left[y_{1}: y_{2}: y_{3}: y_{4}\right]$ on $\mathbb{P}^{3}$ and generic points on $X^{3}$ (those not in $\mathcal{A}^{-1}\left(A^{0} \cup A^{1}\right)$ ) can be described by the homogeneous coordinates $\left[z_{1}: z_{2}: z_{3}: z_{4}\right]$. At these generic points we have $\operatorname{pr}\left(\left[y_{1}: y_{2}: y_{3}: y_{4}\right]\right)=\left[y_{1}: y_{2}: y_{3}: y_{4}\right]$. Since pr is continuous, this implies that at generic points of the fiber $\mathcal{B}^{-1}([1:-1: 1: 1])$, we have

$$
\left[p_{1}: p_{2}\right]=\operatorname{pr}\left(\left[m_{1}: m_{2}: m_{3}\right]\right)=\left[m_{1}: m_{3}\right] \text {. }
$$

In particular, equation (26) places no restriction on the values $\left[p_{1}: p_{2}\right]$, so we conclude that $\operatorname{dim}\left(\operatorname{pr}\left(z_{-,+,+}\right)\right)=1$ and hence that $\operatorname{pr}\left(z_{-,+,+}\right)=\mathcal{A}^{-1}([1:-1: 1: 1])$.

If we repeat the previous calculation over $[1: 1:-1: 1]$, the homogeneous coordinates on $\mathcal{B}^{-1}([1: 1:-1: 1])$ are given by

$$
\begin{aligned}
& \left(\left[y_{1}: y_{2}: y_{3}: y_{4}\right],\left[m_{1}: m_{2}: m_{3}\right]\right) \\
& \quad \text { where }\left(m_{1}, m_{2}, m_{3}\right) \sim\left(y_{1}-y_{4}, y_{2}-y_{4}, y_{3}+y_{4}\right),
\end{aligned}
$$

and again $\left(\left.\widetilde{s}\right|_{\mathcal{B}^{-1}([1: 1:-1: 1])} ^{1}(Z)\right.$ is given at generic points of $\mathcal{B}^{-1}([1: 1:-1: 1])$ by

$$
m_{1}=m_{2} \text {. }
$$

Meanwhile, the fiber $\mathcal{A}^{-1}([1: 1:-1: 1])$ is a result of blowing up the line $\left\{z_{1}=z_{2}=\right.$ $\left.z_{4}\right\} \in A^{1}$. Coordinates in a neighborhood of this fiber are given by

$$
\left(\left[z_{1}: z_{2}: z_{3}: z_{4}\right],\left[p_{1}: p_{2}\right]\right) \quad \text { where }\left(p_{1}, p_{2}\right) \sim\left(z_{1}-z_{4}, z_{2}-z_{4}\right) .
$$

At generic points of the fiber $\mathcal{B}^{-1}([1:-1: 1: 1])$,

$$
\left[p_{1}: p_{2}\right]=\operatorname{pr}\left(\left[m_{1}: m_{2}: m_{3}\right]\right)=\left[m_{1}: m_{2}\right] .
$$

In particular, equation (26) places the restriction $\left[p_{1}: p_{2}\right]=[1: 1]$ on points in $\operatorname{pr}\left(z_{+,-,+}\right)$. We conclude that $\operatorname{dim}\left(\operatorname{pr}\left(z_{+,-,+}\right)\right)=0$.

Using very similar calculations, one finds that the component of $\operatorname{pr}\left(z_{+,+,-}\right)$over $[1$ : $1: 1:-1]$ is a single point on $\mathcal{A}^{-1}([1: 1: 1:-1])$ and that the component of $\operatorname{pr}\left(\mathcal{Z}_{-,-,-}\right)$ over $[1:-1:-1:-1]$ is the whole one-dimensional fiber $\mathcal{A}^{-1}([1:-1:-1:-1])$.

In summary, Proposition 6.6 gives

$$
s^{*}[Z]=[Z]+\left[\mathcal{A}^{-1}([1:-1: 1: 1])\right]+\left[\mathcal{A}^{-1}([1:-1:-1:-1])\right] .
$$

It remains to project $\left[\mathcal{A}^{-1}([1:-1: 1: 1])\right]$ and $\left[\mathcal{A}^{-1}([1:-1:-1:-1])\right]$ back into the basis $\boldsymbol{B}_{2}^{3}$. The divisor $D^{S_{14}}$ is obtained as the blow-up of the proper transform of $\left\{z_{1}=\right.$ $\left.z_{3}=z_{4}\right\}$ within $X_{1}^{3}$. It is biholomorphic to $\mathbb{P}^{1} \times \mathbb{P}^{1}$, with the first factor parameterized by 
TABLE 3. Data for $f_{\rho}^{*}: H^{1,1}\left(X^{2} ; \mathbb{C}\right) \rightarrow H^{1,1}\left(X^{2} ; \mathbb{C}\right)$; the permutation $\rho \in S_{P}$ is given in terms of cycles, an approximate value of the dynamical degree $\lambda_{1}\left(f_{\rho}\right)$ is given as well as the minimal polynomial for $\lambda_{1}\left(f_{\rho}\right)$. There are 120 such maps $f_{\rho}: X^{2} \rightarrow X^{2}$ corresponding to all permutations $\rho \in S_{P}$. The examples in this chart (and all maps which are birationally conjugate to any of these examples) are the only maps $f_{\rho}: X^{2} \rightarrow X^{2}$ for which $\lambda_{1}\left(f_{\rho}\right) \neq 2$.

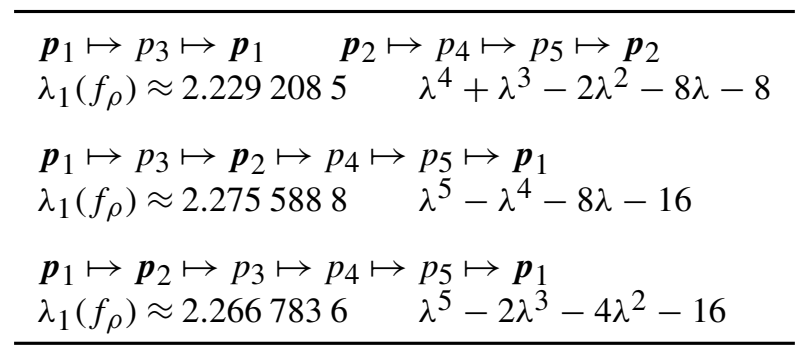

points of the line $z_{1}=z_{3}=z_{4}$ and the second factor parameterized by the fibers of the blow-up. In particular, any two fibers have cohomologous fundamental class. Thus,

$$
\left[\mathcal{A}^{-1}([1:-1: 1: 1])\right] \cong\left[D^{S_{3}} \cap D^{S_{14}}\right]=\left[Z_{14}\right],
$$

because $D^{S_{3}} \cap D^{S_{14}}$ is the fiber of the blow-up over the intersection of the proper transform of $\left\{z_{1}=z_{3}=z_{4}\right\}$ with $E_{[0: 1: 0: 0]}$. Using similar reasoning,

$$
\left[\mathcal{A}^{-1}([1:-1:-1:-1])\right] \cong\left[D^{S_{4}} \cap D^{S_{13}}\right]=\left[Z_{15}\right] .
$$

We summarize our calculation with the following proposition.

Proposition 6.7. With respect to the basis $\boldsymbol{B}_{2}^{3}, s^{*}: H^{2,2}\left(X^{3} ; \mathbb{C}\right) \rightarrow H^{2,2}\left(X^{3} ; \mathbb{C}\right)$ is given by the matrix

$$
s^{*}=\left[\begin{array}{cccccccccccccccc}
4 & 0 & 0 & 0 & 0 & 0 & 0 & 0 & 0 & 0 & 0 & 0 & 0 & 0 & 0 & 0 \\
0 & 4 & 0 & 0 & 0 & 0 & 0 & 0 & 0 & 0 & 0 & 0 & 0 & 0 & 0 & 0 \\
0 & 0 & 4 & 0 & 0 & 0 & 0 & 0 & 0 & 0 & 0 & 0 & 0 & 0 & 0 & 0 \\
0 & 0 & 0 & 4 & 0 & 0 & 0 & 0 & 0 & 0 & 0 & 0 & 0 & 0 & 0 & 0 \\
0 & 0 & 0 & 0 & 4 & 0 & 0 & 0 & 0 & 0 & 0 & 0 & 0 & 0 & 0 & 0 \\
0 & 0 & 0 & 0 & 0 & 4 & 0 & 0 & 0 & 0 & 0 & 0 & 0 & 0 & 0 & 0 \\
0 & 0 & 0 & 0 & 0 & 0 & 4 & 0 & 0 & 0 & 0 & 0 & 0 & 0 & 0 & 0 \\
0 & 0 & 0 & 0 & 0 & 0 & 0 & 4 & 0 & 0 & 0 & 0 & 0 & 0 & 0 & 0 \\
0 & 0 & 0 & 0 & 0 & 0 & 0 & 0 & 4 & 0 & 0 & 0 & 0 & 0 & 0 & 0 \\
0 & 0 & 0 & 0 & 0 & 0 & 0 & 0 & 0 & 4 & 0 & 0 & 0 & 0 & 0 & 0 \\
0 & 0 & 0 & 0 & 0 & 0 & 0 & 0 & 0 & 0 & 4 & 0 & 0 & 0 & 0 & 0 \\
0 & 0 & 0 & 0 & 0 & 0 & 0 & 0 & 0 & 0 & 0 & 2 & 0 & 0 & 0 & 0 \\
0 & 0 & 0 & 0 & 0 & 0 & 0 & 0 & 0 & 0 & 0 & 0 & 2 & 0 & 0 & 0 \\
0 & 0 & 0 & 0 & 0 & 0 & 0 & 0 & 0 & 0 & 0 & 0 & 0 & 2 & 0 & 1 \\
0 & 0 & 0 & 0 & 0 & 0 & 0 & 0 & 0 & 0 & 0 & 0 & 0 & 0 & 2 & 1 \\
0 & 0 & 0 & 0 & 0 & 0 & 0 & 0 & 0 & 0 & 0 & 0 & 0 & 0 & 0 & 1
\end{array}\right] .
$$

\section{Dynamical degree data}

Let $P=\left\{\boldsymbol{p}_{1}, \boldsymbol{p}_{2}, p_{3}, \ldots, p_{n}\right\}$ contain at least three points, and recall that $N:=n-3$. We distinguish the points $\boldsymbol{p}_{1}$ and $\boldsymbol{p}_{2}$ in boldface, as these are precisely the distinguished points in our coordinate system (see $\S 3.1$ ). Using the bases from $\S 6$, we explicitly computed the 
TABLE 4. Data for $f_{\rho}^{*}: H^{1,1}\left(X^{3} ; \mathbb{C}\right) \rightarrow H^{1,1}\left(X^{3} ; \mathbb{C}\right)$ and $f_{\rho}^{*}: H^{2,2}\left(X^{3} ; \mathbb{C}\right) \rightarrow H^{2,2}\left(X^{3} ; \mathbb{C}\right)$; the permutation $\rho \in S_{P}$ is given in terms of cycles, approximate values of the dynamical degrees $\lambda_{1}\left(f_{\rho}\right)$ and $\lambda_{2}\left(f_{\rho}\right)$ are given as well as the minimal polynomials for $\lambda_{1}\left(f_{\rho}\right)$ and $\lambda_{2}\left(f_{\rho}\right)$. There are 720 such maps $f_{\rho}: X^{3} \rightarrow X^{3}$ corresponding to all permutations $\rho \in S_{P}$. The examples in this chart (and all maps which are birationally conjugate to any of these examples) are the only maps $f_{\rho}: X^{3} \rightarrow X^{3}$ for which $\lambda_{1}\left(f_{\rho}\right) \neq 2$, and they are also the only maps $f_{\rho}: X^{3} \rightarrow X^{3}$ for which $\lambda_{2}\left(f_{\rho}\right) \neq 4$.

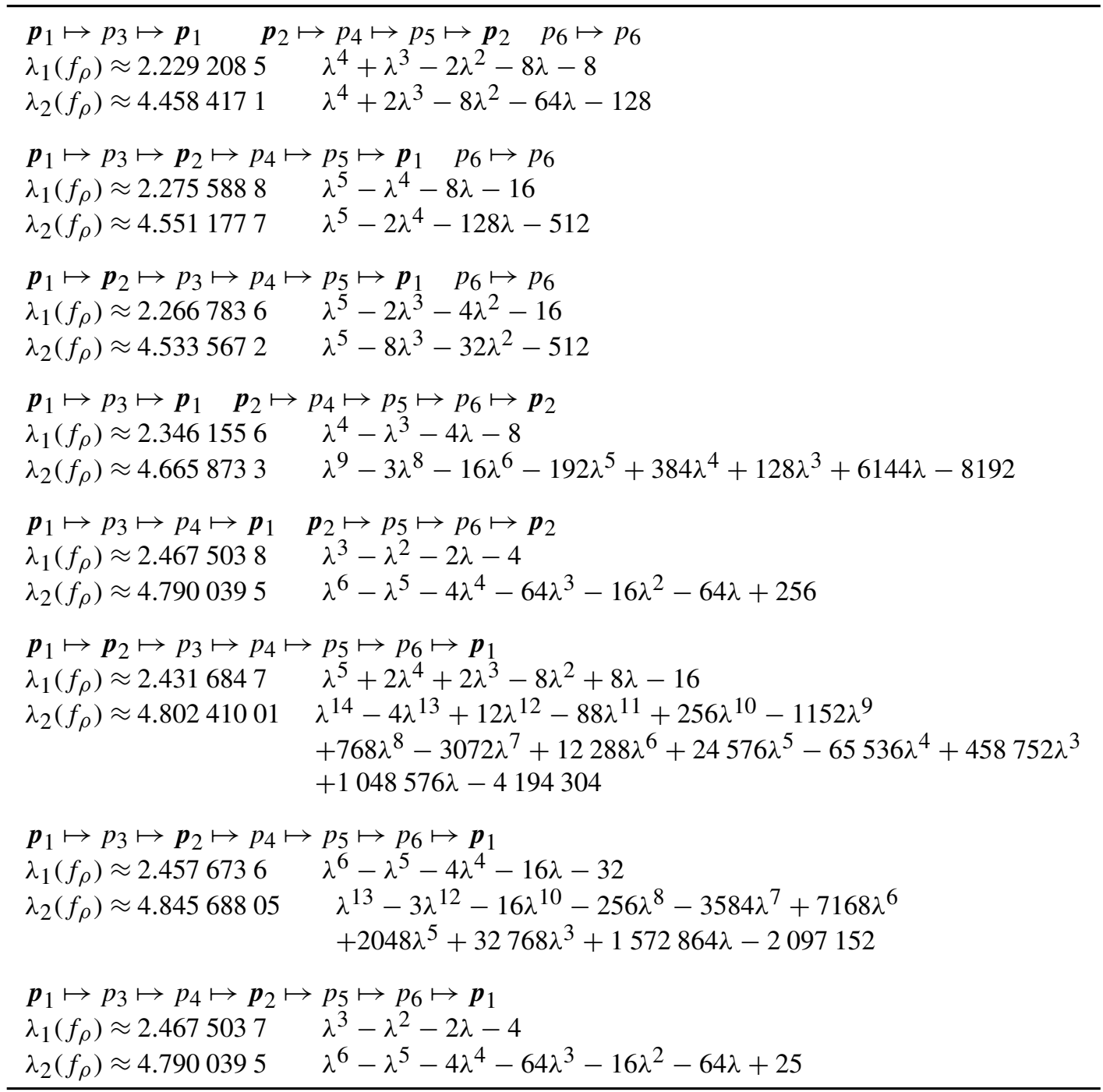

following dynamical degrees:

$|P|=5$ (equivalently $N=2$ ), for all $\rho \in S_{P}$, we compute $\lambda_{1}\left(f_{\rho}\right)$;

$|P|=6$ (equivalently $N=3$ ), for all $\rho \in S_{P}$, we compute $\lambda_{1}\left(f_{\rho}\right)$ and $\lambda_{2}\left(f_{\rho}\right)$;

$|P|=7$ (equivalently $N=4$ ), for all $\rho \in S_{P}$, we compute $\lambda_{1}\left(f_{\rho}\right)$;

$|P|=8$ (equivalently $N=5$ ), for all cyclic permutations $\rho \in S_{P}$, we compute $\lambda_{1}\left(f_{\rho}\right)$.

These are presented in Tables 3-6. As previously mentioned, the methods employed in $\S 6$ should generalize to computing the dynamical degrees $\lambda_{k}\left(f_{\rho}\right)$ for $f_{\rho}: X^{N} \rightarrow X^{N}$ for arbitrary $N$ and $1 \leqslant k \leqslant N$. 
TABLE 5. Data for $f_{\rho}^{*}: H^{1,1}\left(X^{4} ; \mathbb{C}\right) \rightarrow H^{1,1}\left(X^{4} ; \mathbb{C}\right)$; the permutation $\rho \in S_{P}$ is given in terms of cycles, an approximate value of the dynamical degree $\lambda_{1}\left(f_{\rho}\right)$ is given as well as the minimal polynomial for $\lambda_{1}\left(f_{\rho}\right)$. There are 5040 such maps $f_{\rho}: X^{4} \rightarrow X^{4}$ corresponding to all permutations $\rho \in S_{P}$. The examples in this chart (and all maps which are birationally conjugate to any of these examples) are the only maps $f_{\rho}: X^{4} \rightarrow X^{4}$ for which $\lambda_{1}\left(f_{\rho}\right) \neq 2$.

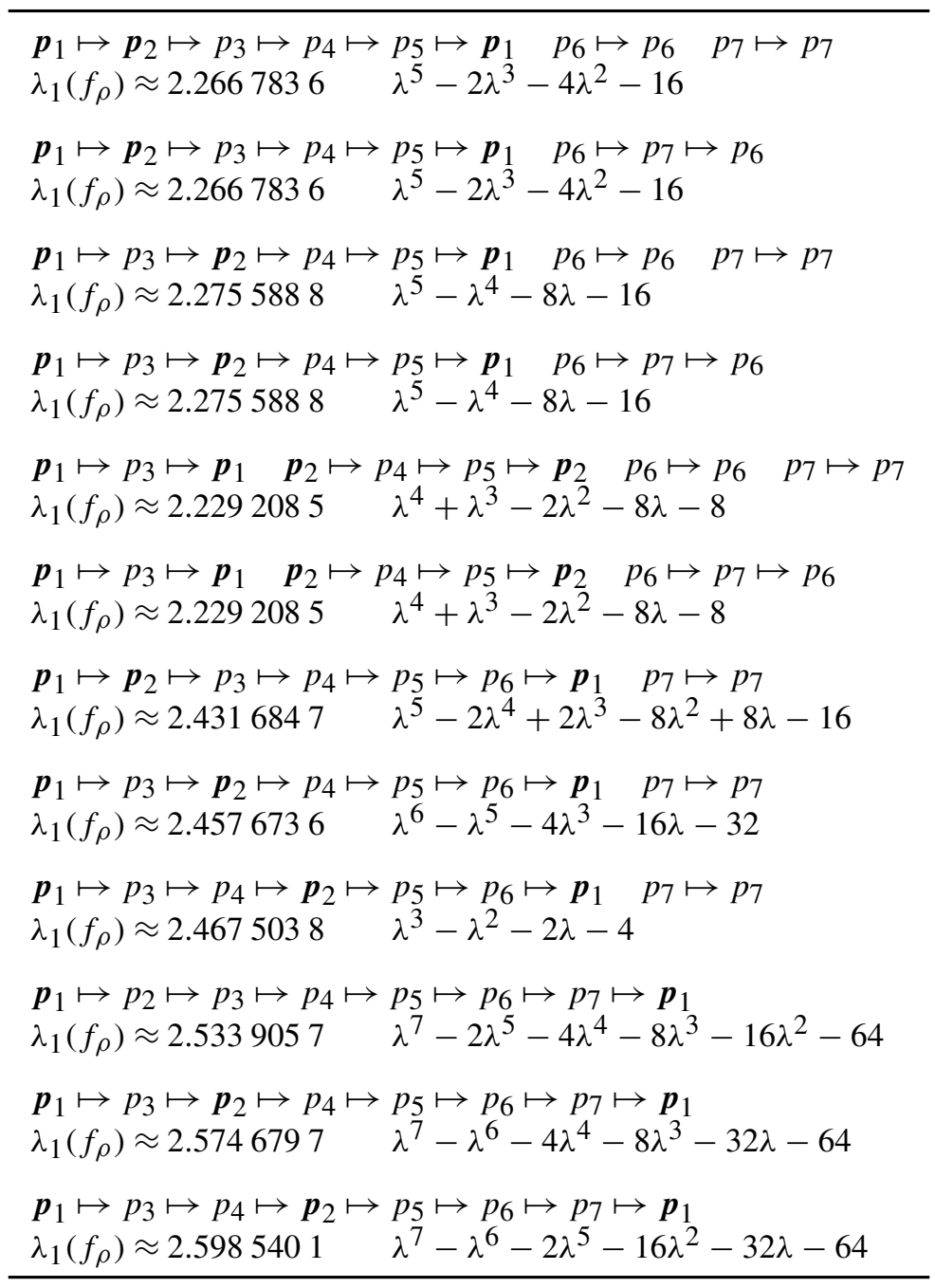

Question 2. To what extent does the structure of the permutation affect the dynamical degrees? There are some patterns that are evident in Tables 3-6. For instance, when $\rho \in S_{P}$ consists of just one cycle of length $n$, the dynamical degrees are 'more complicated' from an algebraic point of view (they tend to have higher algebraic degree). A somewhat related question concerns the characteristic polynomials: in almost all examples, the degree of the eigenvalue corresponding to $\lambda_{k}\left(f_{\rho}\right)$ is strictly less than the dimension of $H^{k, k}\left(X^{N} ; \mathbb{C}\right)$, and the characteristic polynomial factors. What is the dynamical significance of (i) the number of factors, and (ii) the algebraic multiplicity of each factor?

Remark 7.1. One can easily notice from the tables that the first dynamical degree of $f_{\rho}: X^{N} \rightarrow X^{N}$ is always an algebraic integer of degree less than or equal to $N+3$ which is significantly less than $\operatorname{dim}\left(H^{1,1}\left(X^{N} ; \mathbb{C}\right)\right)=2^{N+2}-\left(\begin{array}{c}N+3 \\ 2\end{array}\right)-1$ when $N \geqslant 3$. 
TABLE 6. Data for $f_{\rho}^{*}: H^{1,1}\left(X^{5} ; \mathbb{C}\right) \rightarrow H^{1,1}\left(X^{5} ; \mathbb{C}\right)$; the permutation $\rho \in S_{P}$ is given in terms of cycles, an approximate value of the dynamical degree $\lambda_{1}\left(f_{\rho}\right)$ is given as well as the minimal polynomial for $\lambda_{1}\left(f_{\rho}\right)$. Of the 40320 such maps $f_{\rho}: X^{5} \rightarrow X^{5}$ corresponding to all permutations $\rho \in S_{P}$, we present the data for representative examples corresponding to the cyclic permutations $\rho \in S_{P}$.

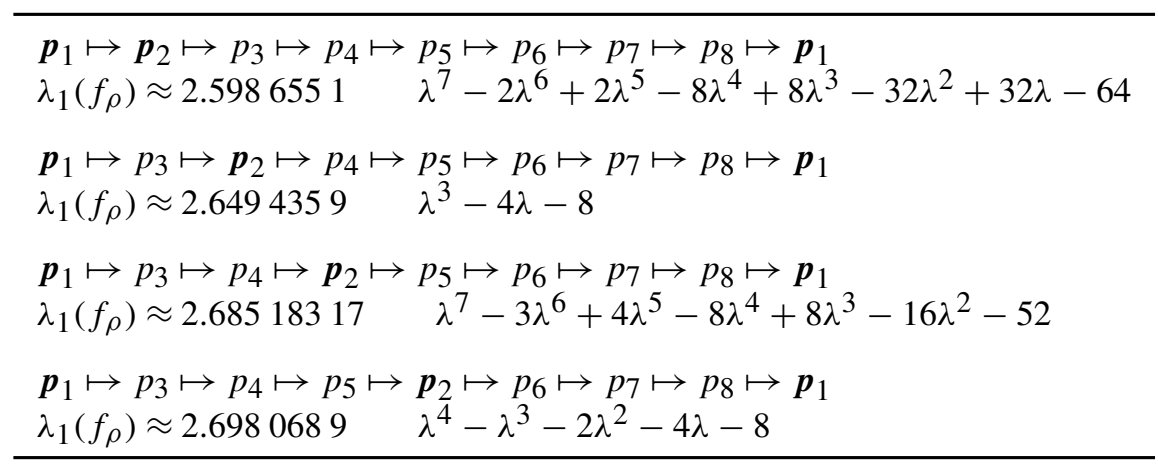

This can be explained as follows. Let $Z^{N}$ be the blow-up of $\mathbb{P}^{N}$ at the $N+2$ points from $A^{0}$, and let $\pi: X^{N} \rightarrow Z^{N}$ be the resulting blow-down map. One can check that $\pi \circ f_{\rho} \circ \pi^{-1}: Z^{N} \rightarrow Z^{N}$ is 1-stable so that its dynamical degree is an algebraic integer of degree less than or equal to $\operatorname{dim}\left(H^{1,1}\left(Z^{N} ; \mathbb{C}\right)\right)=N+3$. The result follows for $f_{\rho}$ since dynamical degrees are invariant under birational conjugacy.

Acknowledgements. We are very grateful to Omar Antolín Camarena for convincing us to use the Sage computer algebra program in our calculations and for helping us to write the scripts. We are also very grateful to Tuyen Truong who informed us of the universal property for blow-ups, which plays a central role in the proof of Theorem 1.2. We have benefited substantially from discussions with Eric Bedford, Xavier Buff, Jeffrey Diller, and Kevin Pilgrim. We also thank the anonymous referee for his or her careful reading of this paper and for making several comments that have improved its exposition.

The research of the first author was supported in part by the NSF DMS-1300315 and the Alfred P. Sloan Foundation. The research of the second author was supported in part by NSF grant DMS-1102597 and start-up funds from the Department of Mathematics at IUPUI.

\section{REFERENCES}

[Am] E. Amerik. A computation of invariants of a rational self-map. Ann. Fac. Sci. Toulouse Math. (6) 18(3) (2009), 445-457.

[B] E. Bedford. The dynamical degrees of a mapping. Proceedings of the International Workshop Future Directions in Difference Equations. Publicacións da Universidade de Vigo, Vigo, Spain, 2011, pp. 3-14.

[BCK] E. Bedford, S. Cantat and K. Kim. Pseudo-automorphisms with no invariant foliation. J. Mod. Dyn. 8(2) (2014), 221-250.

[BK1] E. Bedford and K. Kim. On the degree growth of birational mappings in higher dimension. J. Geom. Anal. 14(4) (2004), 567-596.

[BK2] E. Bedford and K. Kim. Degree growth of matrix inversion: birational maps of symmetric, cyclic matrices. Discrete Contin. Dyn. Syst. 21(4) (2008), 977-1013. 
[BK3] E. Bedford and K. Kim. Dynamics of (pseudo) automorphisms of 3-space: Periodicity versus positive entropy. Publ. Mat. 58(1) (2014), 65-119.

[BT] E. Bedford and T. T. Truong. Degree complexity of birational maps related to matrix inversion. Comm. Math. Phys. 298(2) (2010), 357-368.

[DDG] J. Diller, R. Dujardin and V. Guedj. Dynamics of meromorphic maps with small topological degree I: From cohomology to currents. Indiana Univ. Math. J. 59(2) (2010), 521-561.

[DF] J. Diller and C. Favre. Dynamics of bimeromorphic maps of surfaces. Amer. J. Math. 123(6) (2001), 1135-1169.

[DNT] T.-C. Dinh, V.-A. Nguyen and T. T. Truong. Equidistribution for meromorphic maps with dominant topological degree. Indiana Univ. Math. J., to appear, arXiv:1303.5992.

[DS1] T.-C. Dinh and N. Sibony. Regularization of currents and entropy. Ann. Sci. Éc. Norm. Supér. (4) 37(6) (2004), 959-971.

[DS2] T.-C. Dinh and N. Sibony. Une borne supérieure pour l'entropie topologique d'une application rationnelle. Ann. of Math. (2) 161(3) (2005), 1637-1644.

[DS3] T.-C. Dinh and N. Sibony. Super-potentials of positive closed currents, intersection theory and dynamics. Acta Math. 203(1) (2009), 1-82.

[EH] D. Eisenbud and J. Harris. The Geometry of Schemes (Graduate Texts in Mathematics, 197). Springer, New York, 2000.

[Fa] C. Favre. Les applications monomiales en deux dimensions. Michigan Math. J. 51(3) (2003), 467-475.

[FaW] C. Favre and E. Wulcan. Degree growth of monomial maps and McMullen's polytope algebra. Indiana Univ. Math. J. 61(2) (2012), 493-524.

[Fr] S. Friedland. Entropy of polynomial and rational maps. Ann. of Math. (2) 133(2) (1991), 359-368.

[Fu] W. Fulton. Intersection Theory (Ergebnisse der Mathematik und ihrer Grenzgebiete. 3. Folge. A Series of Modern Surveys in Mathematics [Results in Mathematics and Related Areas. 3rd Series. A Series of Modern Surveys in Mathematics], 2). 2nd edn. Springer, Berlin, 1998.

[GW] U. Görtz and T. Wedhorn. Algebraic Geometry I (Advanced Lectures in Mathematics). Vieweg + Teubner, Wiesbaden, 2010, Schemes with examples and exercises.

[GH] P. Griffiths and J. Harris. Principles of Algebraic Geometry (Wiley Classics Library). John Wiley \& Sons, New York, 1994, reprint of the 1978 original.

[Gu1] V. Guedj. Entropie topologique des applications méromorphes. Ergod. Th. \& Dynam. Sys. 25(6) (2005), 1847-1855.

[Gu2] V. Guedj. Ergodic properties of rational mappings with large topological degree. Ann. of Math. (2) 161(3) (2005), 1589-1607.

[H] R. Hartshorne. Algebraic Geometry (Graduate Texts in Mathematics, 52). Springer, New York, 1977.

[HL] W. J. Harvey and A. Lloyd-Philipps. Symmetry and moduli spaces for Riemann surfaces. Quasiconformal Mappings, Riemann Surfaces, and Teichmüller Spaces (Contemporary Mathematics, 575). American Mathematical Society, 2012, pp. 153-170.

[HP] B. Hasselblatt and J. Propp. Degree-growth of monomial maps. Ergodic Th. \& Dynam. Sys. 27(5) (2007), 1375-1397.

[JW] M. Jonsson and E. Wulcan. Stabilization of monomial maps. Michigan Math. J. 60(3) (2011), 629-660.

[Ka] M. M. Kapranov. Veronese curves and Grothendieck-Knudsen moduli space $\bar{M}_{0, n}$. J. Algebraic Geom. 2(2) (1993), 239-262.

[Ke] S. Keel. Intersection theory of moduli space of stable $n$-pointed curves of genus zero. Trans. Amer. Math. Soc. 330(2) (1992), 545-574.

[Kn] F. Knudsen. The projectivity of the moduli space of stable curves II: The stacks $M_{g, n}$. Math. Scand. 52 (1983), 161-199.

[KM] F. Knudsen and D. Mumford. The projectivity of the moduli space of stable curves I: Preliminaries on 'det' and 'div'. Math. Scand. 39 (1976), 19-55.

[Ko] S. Koch. Teichmüller theory and critically finite endomorphisms. Adv. Math. 248 (2013), 573-617.

[Li1] J.-L. Lin. Pulling back cohomology classes and dynamical degrees of monomial maps. Bull. Soc. Math. France 140(4) (2013, 2012), 533-549.

[Li2] J.-L. Lin. On degree growth and stabilization of three-dimensional monomial maps. Michigan Math. J. 62(3) (2013), 567-579.

[LW] J.-L. Lin and E. Wulcan. Stabilization of monomial maps in higher codimension. Ann. Inst. Fourier (Grenoble) 64(5) (2014), 2127-2146.

[Ll] A. Lloyd-Philipps. Exceptional Weyl groups and complex geometry. PhD Thesis, King's College London, 2007.

[R] R. Roeder. The action on cohomology by compositions of rational maps. Math. Res. Lett. 22(2) (2015), 605-632. 
[RS] A. Russakovskii and B. Shiffman. Value distribution for sequences of rational mappings and complex dynamics. Indiana Univ. Math. J 46(3) (1997), 897-932.

[Si] J. Silverman. Dynamical degree, arithmetic entropy, and canonical heights for dominant rational self-maps of projective space. Ergodic Th. \& Dynam. Sys. 34(2) (2014), 647-678.

[T] T. Terada. Quelques propriétés géométriques du domaine de $f_{1}$ et le groupe de tresses colorées. Publ. RIMS, Kyoto Univ. 17 (1981), 95-111. 